\title{
Ordered categories and additive conjoint measurement on connected sets
}

\author{
D. Bouyssou ${ }^{\mathrm{a}}$, T. Marchant ${ }^{\mathrm{b}}$ \\ ${ }^{a}$ CNRS - LAMSADE, Université Paris Dauphine, F-75775 Paris Cedex 16, \\ France \\ ${ }^{\mathrm{b}}$ Ghent University, Dunantlaan 1, 9000 Ghent, Belgium
}

\begin{abstract}
Suppose that a binary relation is given on a $n$-fold Cartesian product. The study of the conditions guaranteeing the existence of $n$ value functions such that the binary relation can be additively represented is known as additive conjoint measurement. In this paper we analyze a related problem: given a partition of a Cartesian product into $r$ ordered categories, what conditions do ensure the representability of the partition in an additive model?
\end{abstract}

Key words: Classification, conjoint measurement

\section{Introduction}

Suppose we present muli-attributed objects to a subject and we ask him to assign each of them to one among $r$ ordered categories. For example, he may assign sounds to the categories 'high', 'medium' and 'low', students to categories A, B, C, D and E or 10, 9, 8, ..., 0, papers to categories 'accept', 'accept with minor revision', 'major revision' and 'reject', credits to categories 'accept', 'more information needed' and 'reject'. In many circumstances, we can expect that the assignment of an object to a particular category by a subject is determined or at least strongly influenced by its single-attribute characteristics.

Many models have been proposed in the literature for linking the outcome of such experiments to the single-attribute characteristics of the objects. Some of

Email addresses: bouyssou@lamsade.dauphine.fr (D. Bouyssou), thierry.marchant@rug.ac. be (T. Marchant).

Preprint submitted to Journal of Mathematical Psychology 14 May 2008 
them have been axiomatized; for instance, the decomposable model discussed by Goldstein (1991) or the non-compensatory model analysed by Słowiński et al. (2002) and Bouyssou and Marchant (2007a; 2007b). In the decomposable model of Goldstein, there are only two categories ${ }^{1}$ and each level of an attribute is mapped to a real number by a single-attribute value function. An object belongs to the highest category if a monotonic function of all single-attribute values exceeds some threshold. Since the monotonic function is not further specified, it can take many different forms and the decomposable model is therefore very general. It contains, for example, the noncompensatory model but also the additive model, that will be the focus of this paper. In the additive model, the monotonic function is a sum; an object therefore belongs to the highest category if the sum of the single-attribute values exceeds some threshold. Note that this model has a simple geometric interpretation: in the space of the single-attribute values, two consecutive categories are separated by a hyperplane.

The additive model (or some close variants) is very widely used in different domains. In multicriteria decision-aiding, Jacquet-Lagrèze (1995) uses the additive model in his UTADIS technique to help a decision-maker sort alternatives in some ordered categories. In categorization, Reed (1972) discusses the prototype model used to predict the outcome of an experiment where subjects are asked to sort various objects in a fixed number of unordered categories (for a more recent reference, see Stanton et al., 2002). This model assumes the existence of prototypes (one per category) in the multidimensional space where objects are represented. The subject then assigns an object to the category whose prototype is closest to the object. If the distance is Euclidean, the separation between two contiguous categories is then linear, as in the additive model. The multidimensional space in which the objects are represented is sometimes the space of the attributes (this is possible when the attributes are numerical) or sometimes the space resulting from a multidimensional scaling (DeSarbo et al., 1994). In the latter case, the space can eventually be seen as the space of the single-attribute values. Again in categorization, Ashby and Maddox (1993) compare several models, among which the General Linear Classifier. Just like the prototype model, but for different reasons, this model assumes linear frontiers (called decision bounds) between categories. In dataanalysis, the well-know Linear Discriminant Analysis technique also assumes a linear separation between categories (Fisher, 1936; McLachlan, 2004). Note that, when there are only two categories, the difference between ordered and unordered categories vanishes and all the models reviewed in this paragraph are then equivalent to the additive model that we will study in this paper.

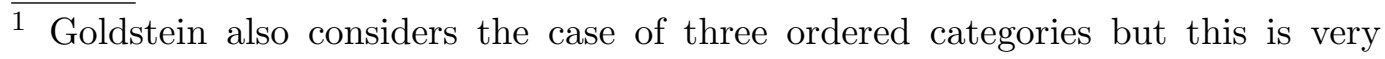
close to the case of two categories because, in Goldstein's model, the intermediate category plays the role of a frontier between the lowest and the highest categories. 
Our analysis will parallel that of preferences in measurement theory and, in particular, in conjoint measurement (e.g. Krantz et al., 1971). Given the outcome of an experiment where a subject has sorted a set $X$ of multi-attributed objects into several ordered categories, we want to find the conditions guaranteeing the existence of single-attribute value functions such that the outcome of the experiment can be represented by the additive model. Such conditions are already known for the decomposable model and the non-compensatory one (see above). For the additive model, they are known only for some special cases. Vind $(1991,2003)$ gives necessary and sufficient conditions when (a) the set $X$ of objects to be sorted is connected, (b) there are at least four attributes, (c) there are exactly two categories and (d) no object is classified in more than one category. In this paper, we will extend Vind's result by weakening assumptions (b), (c) and (d). We will consider that (b') there are at least three attributes, (c') the number of categories is finite and at least two and (d') each object is classified in one category or two consecutive categories, thereby indicating that the object lies at the frontier between two categories. The case of a non-connected set $X$ of objects or of two attributes is addressed in another paper (Bouyssou and Marchant, 2008) because its analysis requires completely different techniques.

Note that the analysis of classification models from a measurement-theoretic perspective is not limited to multi-attributed objects. Nakamura (2004) has axiomatized the partition of a set $X$ of loteries into three classes (worse than, equivalent to, and better than the status quo).

The rest of the paper is organized as follows. The next section introduces the notation and the formal definition of the models that will be analyzed. In Section 3 , we present the axiomatic characterization of the additive model when there are two categories. Section 4 handles the case of more than two categories. The independence of the conditions used in the main result is discussed in Section 5. We then have a short conclusion followed by an appendix with proofs.

\section{Notation and models}

Let $X=X_{1} \times \ldots \times X_{n}$ be a set of objects or stimuli and $N=\{1, \ldots, n\}$ with $n \geq 3$. We also consider $r$ ordered categories denoted by $C^{1}$ (the worst one), $C^{2}, \ldots, C^{r}$ (the best one $)^{2}$. In an experiment, we present each of the objects of $X$ to a subject. For each object $x$, he must tell us to which category $x$ belongs. In some experiments, we may allow that $x$ belongs to two consecu-

$\overline{2}$ In this paper, subscripts are used for attributes and superscripts for categories. A superscript does not indicate an exponentiation. 
tive categories, thereby indicating that $x$ is at the frontier between these two categories. So, our primitives consists of $X$ and $r$ subsets $C^{1}, \ldots, C^{r} \subseteq X$ or, for short, $\left\langle X,\left(C^{k}\right)_{k \in R}\right\rangle$, where $R=\{1, \ldots, r\}$. We will always consider that $C^{k}$ is not empty, for all $k$ in $R$, and that $\left(C^{k}\right)_{k \in R}$ form a covering of $X$, i.e. $\bigcup_{k \in R} C^{k}=X$, or, in some cases, a partition of $X$, i.e. a covering such that $C^{k} \cap C^{k^{\prime}}=\emptyset$ for all $k, k^{\prime} \in R$. An ordered covering of $X$ is a covering such that, for all $k, l \in R,|k-l|>1 \Rightarrow C^{k} \cap C^{l}=\emptyset$. This last condition prevents that non-consecutive categories intersect.

We can now define the models that will be of interest in this paper.

Definition 1 Additive Model with Frontier. The structure $\left\langle X,\left(C^{k}\right)_{k \in R}\right\rangle$ is representable in the Additive model with frontier iff there exist $n$ mappings $u_{i}, i=1 \ldots n: X_{i} \mapsto \mathbb{R}$ and $r+1$ real numbers $s^{1}<\ldots<s^{r+1}$ such that, for all $k \in R$,

$$
x \in C^{k} \Leftrightarrow s^{k} \leq \sum_{i=1}^{n} u_{i}\left(x_{i}\right) \leq s^{k+1} .
$$

Note that an object $x$ can belong to $C^{k-1} \cap C^{k}$ if $\sum_{i=1}^{n} u_{i}\left(x_{i}\right)=s^{k+1}$. We will therefore use the additive model with frontier to represent ordered coverings. In a variant of this model, no object can lie at the frontier between two categories.

Definition 2 Additive Model without Frontier. The structure $\left\langle X,\left(C^{k}\right)_{k \in R}\right\rangle$ is representable in the Additive model without frontier iff there exist $n$ mappings $u_{i}, i=1 \ldots n: X_{i} \mapsto \mathbb{R}$ and $r+1$ real numbers $s^{1}<\ldots<s^{r+1}$ such that, for all $k \in R$,

$$
x \in C^{k} \Leftrightarrow s^{k}<\sum_{i=1}^{n} u_{i}\left(x_{i}\right) \leq s^{k+1} .
$$

This model will be used to represent partitions. Note that we could also permute the strict and the large inequalities in (1). Our choice is arbitrary. All results that will be presented about this model can be easily adapted if one wishes to permute the inequalities. Note that the thresholds $s^{1}$ and $s^{r+1}$ can always be arbirarily set at respectively $-\infty$ and $+\infty$.

In this paper, we will impose that $u_{i}$ be continuous mappings, with respect to a topology that will be introduced later. We will thus speak of continuous representations in the additive model.

Let us introduce some more notation that will soon prove useful. Let $C_{\geq}^{k}=$ $\bigcup_{l \geq k} C^{l}$. The set $C_{\geq}^{k}$ contains the objects that are in category $C^{k}$ or higher. Let $R^{+}=\{2,3, \ldots, r\}$. For all $k \in R, C_{<}^{k}=\bigcup_{l<k} C^{l}$ and $I^{k}=C^{k} \backslash\left(C^{k-1} \cup C^{k+1}\right)$, with the convention that $C^{0}=C^{r+1}=\emptyset$. The set $C_{<}^{k}$ contains the objects 
that are in a category worse than $C^{k}$. Note that $C_{\geq}^{k}$ and $C_{<}^{k}$ are disjoint only when $C^{k} \cap C^{k-1}=\emptyset$. The set $I^{k}$ represents in some sense the interior (not in topological sense) of category $k$. Let also $I_{\geq}^{k}=I^{k} \cup C_{\geq}^{k+1}$.

Let $x$ and $a$ belong to $X$. For any $I \subseteq N,\left(x_{I}, a_{-I}\right)$ denotes an object $z$ such that $z_{i}=x_{i}$ for all $i \in I$ and $z_{i}=a_{i}$ for all $i \in N \backslash I$. When $I$ contains few elements, e.g., $I=\{h, i, j\}$, we just write $\left(x_{h i j}, a_{-h i j}\right)$. The set $\prod_{i \in I} X_{i}$ is denoted by $X_{I}$ and $\prod_{i \in N \backslash I} X_{i}$, by $X_{-I}$.

\section{Two categories}

In this section, we present a set of conditions guaranteeing the existence of an additive representation (with or without frontier) when there are two categories. The first condition depends on a parameter $\nu$ that can vary from 1 to $n$.

A $1 \nu$-Linearity with respect to the Interior or $\nu$-Linearity-I for short. For all $J \subseteq N$ with $\# J=\nu$, all $x_{J}, y_{J} \in X_{J}$, all $a_{-J}, b_{-J} \in X_{-J}$ and all $k, l \in R$,

$$
\left.\begin{array}{c}
\left(x_{J}, a_{-J}\right) \in I_{\geq}^{k} \\
\text { and } \\
\left(y_{J}, b_{-J}\right) \in I_{\geq}^{l}
\end{array}\right\} \Rightarrow\left\{\begin{array}{c}
\left(y_{J}, a_{-J}\right) \in I_{\geq}^{k} \\
\text { or } \\
\left(x_{J}, b_{-J}\right) \in I_{\geq}^{l}
\end{array}\right.
$$

This is a variant of a condition first proposed in Goldstein (1991) for two categories and independently rediscovered (and generalized) in Słowiński et al. (2002). Intuitively, $\nu$-Linearity-I imposes that the elements of $X_{J}$ can be ordered, from best to worse, for any $J \subseteq N$ with $\# J=\nu$. Indeed, if $X_{J}$ is ordered, then one of $x_{J}$ and $y_{J}$ is at least as good as the other one. Given the two premisses in the condition, it is then not possible to have simultaneously $\left(y_{J}, a_{-J}\right)$ in a lower category than $\left(x_{J}, a_{-J}\right)$ and $\left(x_{J}, b_{-J}\right)$ in a lower category than $\left(y_{J}, b_{-J}\right)$.

Let us show the necessity of $\nu$-Linearity-I for the additive model, for any $\nu$. Suppose, contrary to $\nu$-Linearity-I, that

$$
\begin{aligned}
& \left(x_{J}, a_{-J}\right) \in I_{\geq}^{k}, \quad\left(y_{J}, a_{-J}\right) \notin I_{\geq}^{k}, \\
& \left(y_{J}, b_{-J}\right) \in I_{\geq}^{l}, \quad\left(x_{J}, b_{-J}\right) \notin I_{\geq}^{l} .
\end{aligned}
$$


From $\left(x_{J}, a_{-J}\right) \in I_{\geq}^{k}$, we derive

$$
\sum_{i \in J} u_{i}\left(x_{i}\right)+\sum_{i \in N \backslash J} u_{i}\left(a_{i}\right)>s^{k}
$$

From $\left(y_{J}, a_{-J}\right) \notin I_{\geq}^{k}$, we derive

$$
\sum_{i \in J} u_{i}\left(y_{i}\right)+\sum_{i \in N \backslash J} u_{i}\left(a_{i}\right) \leq s^{k}
$$

From these two inequalities, we get $\sum_{i \in J} u_{i}\left(x_{i}\right)>\sum_{i \in J} u_{i}\left(y_{i}\right)$. Similarly, from $\left(y_{J}, b_{-J}\right) \in I_{\geq}^{l}$ and $\left(x_{J}, b_{-J}\right) \notin I_{\geq}^{l}$, we derive $\sum_{i \in J} u_{i}\left(x_{i}\right)<\sum_{i \in J} u_{i}\left(y_{i}\right)$. This is a contradiction. Hence, $\nu$-Linearity-I is a necessary condition for our model.

$\nu$-Linearity-I says nothing about objects that are located on a frontier between two categories. Yet, if we want to use the additive model, these points need to behave consistently with respect to the ordering induced by $\nu$-Linearity-I on $X_{J}$, for any $J \subseteq N$. That is why we introduce a new condition, necessary for the additive model.

A $2 \nu$-Linearity with respect to the Frontier or $\nu$-Linearity-F for short. For all $J \subseteq N$ with $\# J=\nu$, all $x_{J}, y_{J} \in X_{J}$, all $a_{-J}, b_{-J} \in X_{-J}$ and all $k, l \in R^{+}$,

$$
\left.\begin{array}{c}
\left(x_{J}, a_{-J}\right) \in C^{k} \cap C^{k-1} \\
\text { and } \\
\left(y_{J}, b_{-J}\right) \in C^{l} \cap C^{l-1}
\end{array}\right\} \Rightarrow\left\{\begin{array}{c}
\left(y_{J}, a_{-J}\right) \in C_{\geq}^{k} \\
\text { or } \\
\left(x_{J}, b_{-J}\right) \in C_{\geq}^{l}
\end{array}\right.
$$

The intuition behind this condition is the same as behind $\nu$-Linearity-I. The proof of the necessity of this condition, for any $\nu$, is similar to the proof of the necessity of $\nu$-Linearity-I.

The next condition, also depending on a parameter $\nu$ that can vary from 1 to $n$, will tell us more about the structure of the frontier.

A $3 \nu$-Thinness. For all $J \subseteq N$ with $\# J=\nu$ and all $k \in R^{+}$, if there exist $x_{J}, y_{J} \in X_{J}, a_{-J} \in X_{-J}$ such that

$$
\left[\left(x_{J}, a_{-J}\right) \in C^{k-1} \cap C^{k} \text { and }\left(y_{J}, a_{-J}\right) \in C^{k-1} \cap C^{k}\right]
$$

then, for all $b_{-J} \in X_{-J}$ and all $h \in R$,

$$
\left(x_{J}, b_{-J}\right) \in C^{h} \Leftrightarrow\left(y_{J}, b_{-J}\right) \in C^{h} .
$$


We prove its necessity, for all $\nu \in N$. Suppose that $\left(x_{J}, a_{-J}\right)$ and $\left(y_{J}, a_{-J}\right)$ both belong to $C^{k-1} \cap C^{k}$. Then

$$
\sum_{i \in J} u_{i}\left(x_{i}\right)+\sum_{j \in N \backslash J} u_{j}\left(a_{j}\right)=s^{k}=\sum_{i \in J} u_{i}\left(y_{i}\right)+\sum_{j \in N \backslash J} u_{j}\left(a_{j}\right)
$$

and, hence, $\sum_{i \in J} u_{i}\left(x_{i}\right)=\sum_{i \in J} u_{i}\left(y_{i}\right)$. Consequently, if $\left(x_{J}, b_{-J}\right) \in C^{h}$, we have $s^{h} \leq \sum_{i \in J} u_{i}\left(x_{i}\right)+\sum_{j \in N \backslash J} u_{j}\left(b_{j}\right) \leq s^{h+1}$ and, necessarily, $s^{h} \leq \sum_{i \in J} u_{i}\left(y_{i}\right)+$ $\sum_{j \in N \backslash J} u_{j}\left(b_{j}\right) \leq s^{h+1}$, i.e. $\left(y_{J}, b_{-J}\right) \in C^{h}$. Imposing $\nu$-Thinness guarantees that the frontier between any two consecutive categories be 'thin'.

Goldstein (1991) has used variants of 1-Linearity-I, 1-Linearity-F and 1-Thinness for characterizing the decomposable model, i.e. a generalization of the additive model with frontier such that

$$
x \in C^{k} \Leftrightarrow s^{k} \leq F\left(u_{1}\left(x_{1}\right), \ldots, u_{n}\left(x_{n}\right)\right) \leq s^{k+1},
$$

with $F$ strictly increasing in each argument and two categories. In this paper, 1-Linearity-I will not be strong enough: we will need to impose conditions on subsets of cardinality larger than 1 . Note that the same occurs when we try to represent preferences instead of categories (Krantz et al., 1971): independence on singletons is enough for decomposable representations but not for additive ones.

The next condition we present is necessary for any value of $n$ but will be important only when $n=3$.

A 4 Double Cancellation. For all $i, j \in N$ and all $l \in R^{+}$,

$$
\left.\begin{array}{l}
\left(x_{i}, y_{j}, a_{-i j}\right) \in I^{l} \quad \text { and } \\
\left(y_{i}, z_{j}, b_{-i j}\right) \in I^{l} \quad \text { and } \\
\left(z_{i}, x_{j}, c_{-i j}\right) \in I^{l}
\end{array}\right\} \Rightarrow\left\{\begin{array}{l}
\left(y_{i}, x_{j}, a_{-i j}\right) \in C_{\geq}^{l} \text { or } \\
\left(z_{i}, y_{j}, b_{-i j}\right) \in C_{\geq}^{l} \text { or } \\
\left(x_{i}, z_{j}, c_{-i j}\right) \in C_{\geq}^{l} .
\end{array}\right.
$$

Although this may not be apparent at first sight, it plays the same role as double cancellation (see e.g. Krantz et al., 1971) or the Thomsen condition in conjoint measurement based on a binary relation. That is why we also call it Double Cancellation.

The necessity of this axiom is easy to show. Assume the three premisses hold and none of the consequences. We derive

$$
\begin{aligned}
& u_{i}\left(x_{i}\right)+u_{j}\left(y_{j}\right)+\sum_{h \in N \backslash\{i, j\}} u_{h}\left(a_{h}\right)>u_{i}\left(y_{i}\right)+u_{j}\left(x_{j}\right)+\sum_{h \in N \backslash\{i, j\}} u_{h}\left(a_{h}\right), \\
& u_{i}\left(y_{i}\right)+u_{j}\left(z_{j}\right)+\sum_{h \in N \backslash\{i, j\}} u_{h}\left(b_{h}\right)>u_{i}\left(z_{i}\right)+u_{j}\left(y_{j}\right)+\sum_{h \in N \backslash\{i, j\}} u_{h}\left(b_{h}\right) .
\end{aligned}
$$




$$
u_{i}\left(z_{i}\right)+u_{j}\left(x_{j}\right)+\sum_{h \in N \backslash\{i, j\}} u_{h}\left(c_{h}\right)>u_{i}\left(x_{i}\right)+u_{j}\left(z_{j}\right)+\sum_{h \in N \backslash\{i, j\}} u_{h}\left(c_{h}\right) .
$$

If we add these three inequalities and perform some simplifications, we find $0>0$, a contradiction.

All conditions presented up to this point are necessary for the existence of an additive representation but are not sufficient if we do not impose some structural conditions on $X$ (just like with additive representations of preferences). The main structural condition is connectedness. In order to formally state this condition, we first need to define the binary relation $\succsim_{I}^{R}$, for each subset of dimensions $I \subset N$, and the interval topology relative to $\succsim_{i}^{R}$.

Let $x_{I} \succsim_{I}^{R} y_{I}$ iff, $\forall a_{-I} \in X_{-I}, \forall k \in R$,

- $\left(y_{I}, a_{-I}\right) \in C^{k} \cap C^{k-1}$ implies $\left(x_{I}, a_{-I}\right) \in C_{\geq}^{k}$ and

- $\left(y_{I}, a_{-I}\right) \in I^{k}$ implies $\left(x_{I}, a_{-I}\right) \in I_{\geq}^{k}$.

When $I=\{i\}$, we just write $\succsim_{i}^{R}$. By construction, $\succsim_{I}^{R}$ is reflexive and transitive for any $I \subset N$. The interval topology on $X_{i}$ induced by $\succsim_{i}^{R}$ is generated by taking the sets $\left\{z_{i} \in X_{i}: z_{i} \succsim_{i}^{R} x_{i}\right\}$ and $\left\{z_{i} \in X_{i}: x_{i} \succsim_{i}^{R} z_{i}\right\}$ to be a subbasis for closed sets. The closed sets in this topology are thus the unions of finite intersections of sets belonging to the subbasis. In the sequel, we will impose that $X_{i}$ be connected in the interval topology induced by $\succsim_{i}^{R}$. Let us turn to a new condition to be used only with the additive model with frontier.

A 5 Restricted Solvability. For all $i \in N$, all $a_{-i} \in X_{-i}$ and all $k \in R^{+}$, if there are $x_{i}$ and $y_{i}$ such that $\left(x_{i}, a_{-i}\right) \in C^{k-1}$ and $\left(y_{i}, a_{-i}\right) \in C^{k}$, then there exists $z_{i}$ such that $\left(z_{i}, a_{-i}\right)$ belongs to $C^{k-1} \cap C^{k}$.

Restricted Solvability is not a necessary condition for the additive model with frontier in general but, if we want to use it with sets $X_{i}$ connected in the interval topology induced by $\succsim_{i}^{R}$ and if we want that $u_{i}$ be continuous with respect to the interval topology, then it is necessary. Indeed, suppose $\left(x_{i}, a_{-i}\right) \in I^{k-1}$ and $\left(y_{i}, a_{-i}\right) \in I^{k}$. Because we assume the existence of an additive representation with frontier, we find

$$
u_{i}\left(x_{i}\right)+\sum_{j \in N \backslash\{i\}} u_{j}\left(a_{j}\right)<s^{k}
$$

and

$$
u_{i}\left(y_{i}\right)+\sum_{j \in N \backslash\{i\}} u_{j}\left(a_{j}\right)>s^{k} .
$$

There exists $\rho \in \mathbb{R}: \rho+\sum_{j \in N \backslash\{i\}} u_{j}\left(a_{j}\right)=s^{k}$. Define two sets $V$ and $W$ by $V=\left\{z_{i} \in X_{i}: u_{i}\left(z_{i}\right)<\rho\right\}$ and $W=\left\{z_{i} \in X_{i}: u_{i}\left(z_{i}\right)>\rho\right\}$. These two sets are clearly open (because $u_{i}$ is continuous) and disjoint. Suppose, in contradiction to Restricted Solvability, that there is no $z_{i}$ such that $\left(z_{i}, a_{-i}\right)$ 
belongs to $C^{k-1} \cap C^{k}$. So, $W \cup V=X_{i}$ and, hence, $W$ and $V$ form a partition of $X_{i}$. This contradicts the connectedness of $X_{i}$.

Most representation theorems about additive representations on connected sets do not use any form of solvability. Solvability is usually used only in theorems where no topological condition is assumed. In our case, when there are only two categories, we could replace Restricted Solvability by the constraint that $I^{1}$ and $I^{2}$ be open. Indeed, if $I^{1}$ and $I^{2}$ are open, then Restricted Solvability necessarily holds, as shown hereunder. Define $M\left(a_{-i}\right)=\left\{x_{i} \in X_{i}\right.$ : $\left.\left(x_{i}, a_{-i}\right) \in I^{1}\right\}$ and $N\left(a_{-i}\right)=\left\{x_{i} \in X_{i}:\left(x_{i}, a_{-i}\right) \in I^{2}\right\}$. The sets $I^{1}$ and $I^{2}$ being open, $M\left(a_{-i}\right)$ and $N\left(a_{-i}\right)$ are also open. If one of these sets is empty, then Restricted Solvability trivially holds for $a_{-i}$. If they are not empty, then, $X_{i}$ being connected, $M\left(a_{-i}\right)$ and $N\left(a_{-i}\right)$ cannot be a partition of $X_{i}$. By construction, $M\left(a_{-i}\right) \cap N\left(a_{-i}\right)$ is empty. Hence, there is some $x_{i}$ such that $\left(x_{i}, a_{-i}\right) \notin I^{1}$ and $\left(x_{i}, a_{-i}\right) \notin I^{2}$. This implies $\left(x_{i}, a_{-i}\right) \in C^{1} \cap C^{2}$ and proves that Restricted Solvability holds for $a_{-i}$. If we follow the same reasoning with all $i \in N$ and all $a_{-i} \in X_{-i}$, we find that Restricted Solvability holds.

We say that a dimension $i$ is influent if there are $x_{i}, y_{i} \in X_{i}$ such that $x_{i} \succ_{i}^{R}$ $y_{i}$. If some dimensions are not influent, then these can be dropped and the representation is analysed in a space with fewer dimensions.

In order to avoid trivial cases, we will restrict our attention to non-degenerate coverings, i.e. such that $I^{k} \neq \emptyset$ for all $k \in R$. Our main result for the case of two categories is the following.

Theorem 1 Consider a structure $\left\langle X,\left(C^{1}, C^{2}\right)\right\rangle$ with $n \geq 3$ and $\left(C^{1}, C^{2}\right)$ a non-degenerate covering of $X$ such that $\left\langle X_{i}, \succsim_{i}^{R}\right\rangle$ is influent and connected in the interval topology induced by $\succsim_{i}^{R}$, for every $i \in N$. Then it has a continuous representation in the Additive Model With Frontier iff it satisfies 1-LinearityI, 1-Linearity-F, 1-Thinness, Restricted Solvability and one of the following conditions: 2-Linearity-I (when $n>3$ ) or Double Cancellation (when $n=3$ ). The mappings $u_{i}$ are unique up to $n$ positive affine transformations with the same multiplicative constant

The actual proof of Th. 1 (and of all other results) is deferred to the appendix but we give here a sketch of it. Using 1-Linearity-I, 1-Linearity-F, 1-Thinness and Restricted Solvability, it is not hard to find that the covering admits a strictly increasing decomposable representation as in (2), as shown in Goldstein (1991). In this numerical representation, $u\left(C^{1} \cap C^{2}\right)$, i.e. the image of the frontier, is a strictly monotonic hypersurface in $u(X)$. The intersection of this hypersurface with $u\left(x_{1} \times X_{-1}\right)$ is another hypersurface of dimension $n-1$. If we let $x_{1}$ vary, we then obtain a collection of hypersurfaces that can be seen as equivalence classes of a binary relation $\succsim^{*}$ on a subset of $X_{-1}$. We then show that the existence of an additive representation of $\succsim^{*}$ is equivalent to the ex- 
istence of an additive representation of the covering (thanks to 2-Linearity-I or Double Cancellation). Finally, the conditions guaranteeing the existence of an additive representation of $\succsim^{*}$ can be found in a result of Chateauneuf and Wakker (1993). The reason why we use this result and not a more standard one is that $\succsim^{*}$ is not defined on a Cartesian product. The independence of the conditions of Theorem 1 is analyzed in Section 5 .

Until now, we only considered the representation of ordered coverings. The representation of partitions is very similar. The differences are:

- 1-Linearity-F and 1-Thinness are superfluous with partitions because they are trivially satisfied.

- Restricted Solvability is not satisfied by partitions since no point can lie in $C^{1} \cap C^{2}$.

Instead of Restricted Solvability, we impose that $C^{2}$ be open and we now show that this condition is necessary. The mappings $u_{i}$ being continuous, the mapping $\sum_{i \in N} u_{i}$ is also continuous. The image of $C^{2}$ through this mapping is open; indeed, it is represented by a strict inequality $\left(\sum_{i \in N} u_{i}>s^{1}\right)$. Hence, the inverse image, i.e. $C^{2}$, is also open.

We then obtain the following corollary of Theorem 1.

Corollary 1 Consider a structure $\left\langle X,\left(C^{1}, C^{2}\right)\right\rangle$ with $n \geq 3$ and $\left(C^{1}, C^{2}\right)$ a partition of $X$ such that $\left\langle X_{i}, \succsim_{i}^{R}\right\rangle$ is influent and connected in the interval topology induced by $\succsim_{i}^{R}$, for every $i \in N$. Then it has a continuous representation in the Additive Model Without Frontier iff it satisfies 1-Linearity-I, 2-Linearity-I (when $n>3$ ), Double Cancellation (when $n=3$ ) and $C^{2}$ is open in the product topology generated by the interval topology on each $X_{i}$. The mappings $u_{i}$ are unique up to $n$ positive affine transformations with the same multiplicative constant.

This corollary is a generalization of Theorem 11 of Vind (2003), also presented at p. 122 of Vind (1991). Vind did not use Double Cancellation and did thus not handle representation problems with $n=3$. In the next section, we will further generalize Vind's result by considering partitions with more than two categories.

\section{More than two categories}

We shortly explain how we will go from 2 to $r$ categories. For $k \in R^{+}$, let

$$
X_{i}^{k}=\left\{x_{i} \in X_{i}:\left(x_{i}, x_{-i}\right) \in C^{k} \cap C^{k-1} \text { for some } x_{-i} \in X_{-i}\right\}
$$




$$
\begin{gathered}
X^{k}=X_{1}^{k} \times \ldots \times X_{n}^{k} \\
C^{k, 2}=\left\{x \in X^{k}: x \in C_{\geq}^{k}\right\} \text { and } C^{k, 1}=\left\{x \in X^{k}: x \in C_{<}^{k}\right\} .
\end{gathered}
$$

The sets $C^{k, 2}$ and $C^{k, 1}$ clearly form a covering of $X^{k}$. So, if each covering $\left(C^{k, 2}, C^{k, 1}\right)_{k \in R^{+}}$satisfies the conditions of Theorem 1 , we can construct an additive representation for each covering. But these $r-1$ representations will be independent of each other. For the points lying in $X^{k} \cap X^{l}, k \neq l$, it is not sure that the additive representation based on $X^{k}$ will be compatible with the one based on $X^{l}$. If we want to have one representation for $\left(C^{k}\right)_{k \in R}$ instead of $r-1$ representations, we have to impose the following additional condition, guaranteeing that everything goes well for the points lying in $X^{k} \cap X^{l}$ :

A 6 Overlap. There is $l \in R^{+}$such that $X=X^{l}$ and, for all $k \in R^{+} \backslash\{l\}$ and all $i \in N$, there is $x^{(i)} \in C^{k-1} \cap C^{k}$ such that $\left(x_{-i}^{(i)}, x_{i}^{\prime}\right) \in C^{l-1} \cap C^{l}$ for some $x_{i}^{\prime} \in X_{i}$.

Unfortunately, Overlap is not a necessary condition for the additive model as shown in the following example.

Example 1 Let $X=[0,3 / 2] \times[0,1] \times[0,1]$. Let $x \in C^{1}$ iff $x_{1}+x_{2}+x_{3} \leq 1$. Let $x \in C^{2}$ iff $1 \leq x_{1}+x_{2}+x_{3} \leq 5 / 2$. Let $x \in C^{3}$ iff $5 / 2 \leq x_{1}+x_{2}+x_{3}$. This covering clearly has an additive representation but does not satisfy Overlap. Indeed, $X^{2} \neq X \neq X^{3}$.

We are now ready to state the main result of this section.

Theorem 2 Consider a structure $\left\langle X,\left(C^{k}\right)_{k \in R}\right\rangle$ with $n \geq 3$ and $\left(C^{k}\right)_{k \in R}$ a non-degenerate overlapping covering of $X$ such that $\left\langle X_{i}, \succsim_{i}^{R}\right\rangle$ is influent and connected in the interval topology induced by $\succsim_{i}^{R}$, for every $i \in N$. Then it has a continuous representation in the Additive Model With Frontier iff it satisfies 1-Linearity-I, 1-Linearity-F, 1-Thinness, 2-Linearity-I, Restricted Solvability and one additional condition when $n=3$ : Double Cancellation. The mappings $u_{i}$ are unique up to $n$ positive affine transformations with the same multiplicative constant.

Without Overlap, the uniqueness of the representation is no longer guaranteed: the representations on the different $X^{k}$ can be independent of each other. With Overlap, the different representations are linked and 2-Linearity-I imposes that the hyperplanes separating the categories be parallel to each other.

When we have a partition, 1-Thinness and 1-Linearity-F are trivially satisfied. We therefore have the following corollary to Theorem 2.

Corollary 2 Consider a structure $\left\langle X,\left(C^{k}\right)_{k \in R}\right\rangle$ with $n \geq 3$ and $\left(C^{k}\right)_{k \in R}$ an overlapping partition of $X$ such that $\left\langle X_{i}, \succsim_{i}^{R}\right\rangle$ is influent and connected in 
the interval topology induced by $\succsim_{i}^{R}$, for every $i \in N$. Then it has a continuous representation in the Additive Model Without Frontier iff it satisfies 1-Linearity-I, 2-Linearity-I, Double Cancellation (when $n=3$ ) and $C_{\geq}^{k}$ is open for all $k \in R^{+}$in the product topology generated by the interval topology on each $X_{i}$. The mappings $u_{i}$ are unique up to $n$ positive affine transformations with the same multiplicative constant.

\section{Independence of the conditions}

The following examples prove that the conditions of Theorem 1 are independent when $n=3$.

Example 2 Let $X=] 0,1[\times] 0,1[\times] 0,1\left[\right.$. Let $x \in C^{1}$ iff $x_{1}+x_{2}+x_{3} \leq 1 / 2$ or $x_{1}+x_{2}+x_{3}=1$. Let $x \in C^{2}$ iff $x_{1}+x_{2}+x_{3}>1 / 2$. A routine check shows that this structure satisfies 1-Linearity-F, 1-Thinness, Double Cancellation and Restricted Solvability. But 1-Linearity-I fails with $x_{1}=1 / 2, y_{1}=1 / 4, a_{2}=$ $1 / 4, b_{2}=1 / 2, a_{3}=0, b_{3}=0$.

Example 3 Let $X=] 0,1[\times] 0,1[\times] 0,1\left[\right.$. Let $x \in C^{1}$ iff $x_{1}+x_{2}+x_{3} \leq 3 / 2$ and $x \in C^{2}$ iff $\left[x_{1}+x_{2}+x_{3}=1\right.$ or $\left.x_{1}+x_{2}+x_{3}>3 / 2\right]$. A routine check shows that this structure satisfies 1-Linearity-I, 1-Thinness, Double Cancellation and Restricted Solvability. But it does not satisfy 1-Linearity-F. Indeed, $(1 / 3,1 / 3,1 / 3) \in C^{1} \cap C^{2}$ and $(1 / 2,1 / 4,1 / 4) \in C^{1} \cap C^{2}$ but $(1 / 3,1 / 4,1 / 4) \in I^{1}$ and $(1 / 2,1 / 3,1 / 3) \in I^{1}$.

Example 4 Let $X=] 0,1[\times] 0,1[\times] 0,1\left[\right.$. Let $x \in C^{1}$ iff $x_{1}+x_{2}+x_{3} \leq 2$ and $x \in C^{2}$ iff $x_{1}+x_{2}+x_{3} \geq 1$. A routine check shows that this structure satisfies 1-Linearity-I, 1-Linearity-F, Double Cancellation and Restricted Solvability. But it does not satisfy 1-Thinness. Indeed, $(1 / 2,1 / 2,1 / 2) \in C^{1} \cap C^{2}$ and $(1 / 4,1 / 2,1 / 2) \in C^{1} \cap C^{2}$ but $(1 / 2,1,5 / 8) \in I^{2}$ and $(1 / 4,1,5 / 8) \in C^{1} \cap C^{2}$.

Example 5 Let $X=] 0,1[\times] 0,1[\times] 0,1\left[\right.$. Let $x \in C^{1}$ iff $x_{1}+x_{2}+x_{3} \leq 1$ and $x \in C^{2}$ iff $x_{1}+x_{2}+x_{3}>1$. A routine check shows that this structure satisfies 1-Linearity-I, 1-Linearity-F, 1-Thinness and Double Cancellation. But it obviously violates Restricted Solvability.

Example 6 Let $X=\mathbb{R} \times \mathbb{R} \times \mathbb{R}$. Let $x \in C^{1}$ iff $x_{1}+x_{2}+x_{3}+\min \left(x_{1}, x_{2}\right) \leq$ $13 / 10$ and $x \in C^{2}$ iff $x_{1}+x_{2}+x_{3}+\min \left(x_{1}, x_{2}\right) \geq 13 / 10$. It is easy to check that this structure satisfies 1-Linearity-I, 1-Linearity-F, 1-Thinness and Restricted Solvability. But Double Cancellation fails with $x_{1}=1 / 3, y_{1}=0, z_{1}=2 / 3, x_{2}=$ $7 / 8, y_{2}=1 / 3, z_{2}=3 / 2, a_{3}=1 / 3, b_{3}=-1 / 6, c_{3}=-7 / 8$. 
When $n \geq 4$, it is easy to adapt examples $3-5$. We also provide an example showing that 2-Linearity-I is not implied by the other conditions.

Example 7 Let $X=\mathbb{R}_{+} \times \mathbb{R}_{+} \times \mathbb{R}_{+} \times \mathbb{R}_{+}$. Let $x \in C^{1}$ iff $x_{1} x_{2}+x_{3} x_{4} \leq 70$ and $x \in C^{2}$ iff $x_{1} x_{2}+x_{3} x_{4} \geq 70$. It is easy to check that this structure satisfies 1-Linearity-I, 1-Linearity-F, 1-Thinness and Restricted Solvability. But it does not satisfy 2-Linearity-I. Indeed, $(3,5,7,8) \in I^{2}$ and $(7,9,4,5) \in I^{2}$ but $(3,9,7,5) \in I^{1}$ and $(7,5,4,8) \in I^{1}$.

Unfortunately, we did not succeed in finding an example proving that 1Linearity-I is not implied by the other conditions. So, the issue of the independence of the conditions of Theorem 1 when $n \geq 4$ is not completely solved.

There is an interesting result in Vind (1991) about the independence of the conditions. We present it using our notation.

Theorem 3 [Theorem 2 in Vind (1991)] Consider a structure $\left\langle X,\left(C^{1}, C^{2}\right)\right\rangle$ with $\left(C^{1}, C^{2}\right)$ a partition of $X$. Assume each dimension is influent, $C^{2}$ is open and the structure $\left\langle X,\left(C^{1}, C^{2}\right)\right\rangle$ is linear-I on two sets of dimensions $I$ and $J$, with $X_{I \cap J}, X_{I \backslash J}, X_{J \backslash I}, X_{\bar{I} \cap \bar{J}}$ all connected and $I \cap J, I \backslash J, J \backslash I, \bar{I} \cap \bar{J}$ all non-empty. Then the structure $\left\langle X,\left(C^{1}, C^{2}\right)\right\rangle$ is linear-I on all sets of the field generated by $I$ and $J$.

This theorem implies, in particular, that, 1-Linearity-I can be dropped in Corollary 2 (and probably also in Theorem 1) since any singleton is the intersection of two pairs. So, it seems that this result solves the question of the independence of the conditions. Yet a crucial point appears mysterious to us in Vind's proof of Theorem 3 and we therefore prefer not to use it. Indeed, in the proof, Vind writes (with our notation) 'When $\bar{I} \cap \bar{J} \neq \emptyset$, there is on $X_{I \cup J}$ an order which is ...' But, according to us, if we do not impose 1-linearity-I on $I \cup J$ (and Vind does not in Theorem 3), we do not know if the relation defined on $X_{I \cup J}$ by Vind on p. 121 is transitive. So, it might not be an order and, in that case, the proof collapses.

\section{Conclusion}

We have provided an axiomatic characterization of the additive model for ordered categories when the set $X$ is connected. This permits us to have a deeper understanding of the additive model. It can help us elicit the parameters of the additive model and also test whether a given ordered classification is representable by the additive model. 
One rather surprising feature of our results is that additiv erepresentations with strong uniqueness properties can be obtained on the basis of much poorer information than what is traditionally used in conjoint measurement.

Yet some problems remain open. For example, are the axioms in Theorem 1 independent? Is it possible to replace the connectedness condition by an Archimedean one thereby using only algebraic conditions ${ }^{3}$ ? In Bouyssou and Marchant (2008), we treated this problem but under the assumption that, for all $i$ and all $a_{-i} \in X_{-i}$, there is $x_{i}$ such that $\left(x_{i}, a_{-i}\right) \in C^{1} \cap C^{2}$ (unrestricted solvability). It would of course be interesting to solve this problem under the weaker assumption of Restricted Solvability. Note that the case where $X$ is finite has been treated in Bouyssou and Marchant (2008), without any solvability.

How can we deal with unordered categories separated by line segments, as in the prototype model? Concerning this last question, let us mention an interesting paper about the axiomatization of a variant of the prototype model (Azrieli and Lehrer, 2007). This paper handles the case of a finite number (possibly larger than 2) of unordered categories but there are important differences with respect to our problem. Indeed, Azrieli and Lehrer impose that each attribute consists of the set of real numbers and that the distances between prototypes and objects are computed in the space made of the Cartesian product of the attributes and not in the space of the single-attribute values. These two restrictions make the problem quite different from the one studied here.

\section{A Proofs}

We say that $X_{i}$ is unbounded if there are no $t_{i} \in X_{i}: t_{i} \succsim_{i}^{R} w_{i}, \forall w_{i} \in X_{i}$ and no $b_{i} \in X_{i}: w_{i} \succsim_{i}^{R} b_{i}, \forall w_{i} \in X_{i}$. Note that unboundedness implies influence but the converse is not true. Let us replace influence by the stronger unboundedness in the statement of Th. 1; we obtain the following proposition.

Proposition 1 Consider a structure $\left\langle X,\left(C^{1}, C^{2}\right)\right\rangle$ with $n \geq 3$ and $\left(C^{1}, C^{2}\right)$ a non-degenerate covering of $X$ such that $X_{i}$ is unbounded and connected in the interval topology induced by $\succsim_{i}^{R}$, for every $i \in N$. Then it has a continuous representation in the Additive Model With Frontier iff it satisfies 1-LinearityI, 1-Linearity-F, 1-Thinness, Restricted Solvability and one of the following conditions: 2-Linearity-I (when $n>3$ ) or Double Cancellation (when $n=3$ ). The mappings $u_{i}$ are unique up to $n$ positive affine transformations with the

$\overline{3}$ Wakker (1988) has shown that algebraic conditions are weaker than topological ones when dealing with preferences expressed by means of a preference relation. 
same multiplicative constant.

We will prove this proposition before proving Th. 1. But, first, a series of lemmas.

\section{A.1 Preliminary lemmas}

Most of the lemmas that follow are stated for two categories only because they will be used only in the proof of Proposition 1. Yet some other lemmas are stated for $r$ categories because they will also be used later.

Lemma 1 If $\left\langle X,\left(C^{k}\right)_{k \in R}\right\rangle$ satisfies Restricted Solvability, then $\left(x_{I}, a_{-I}\right) \in I_{\geq}^{k}$ and $\left(x_{I}, b_{-I}\right) \notin C_{\geq}^{k}$ imply that there is $c_{-I}$ such that $\left(x_{I}, c_{-I}\right) \in C^{k-1} \cap C^{k}$.

Proof. Consider $c_{-I}^{(1)}$ defined by $c_{-I}^{(1)}=\left(a_{-I j}, b_{j}\right)$ for some $j \notin I$. Three cases are possible.

(1) $\left(x_{I}, c_{-I}^{(1)}\right) \in C^{k-1} \cap C^{k}$ and, letting $c_{-I}=c_{-I}^{(1)}$, we are done.

(2) $\left(x_{I}, c_{-I}^{(1)}\right) \notin C_{\geq}^{k}$. By Restricted Solvability, there is $z_{j}$ such that $\left(x_{I}, c_{-I j}^{(1)}, z_{j}\right) \in$ $C^{k-1} \cap C^{k}$ and, letting $c_{-I}=\left(c_{-I j}^{(1)}, z_{j}\right)$, we are done.

(3) $\left(x_{I}, c_{-I}^{(1)}\right) \in I_{\geq}^{k}$. We then define $c_{-I}^{(2)}$ letting $c_{-I}^{(2)}=\left(a_{-I j l}, b_{j}, b_{l}\right)$ for some $l \in N \backslash(I \cup\{j\})$. We distinguish again the same three cases and, eventually, construct $c_{-I}^{(3)}$. This process continues until we find an element in $C^{k-1} \cap C^{k}$. It necessarily stops before we construct $c_{-I}^{(m)}$, with $m=$ $\#(N \backslash I)$, because the only possibility with $c_{-I}^{(m-1)}$ is case (2).

Note that $I$ can be empty in this lemma.

Lemma 2 If $\left\langle X,\left(C^{k}\right)_{k \in R}\right\rangle$ satisfies Restricted Solvability, $\nu$-Linearity-F, $\nu$ Linearity-I and $\nu$-Thinness, then $\succsim_{I}^{R}$ is a weak order, for every $I \subset N$ such that $\# I=\nu$.

Proof. Since $\succsim_{I}^{R}$ is transitive by definition, we just need to prove that it is also complete. Suppose, on the contrary, that $x_{I} \chi_{I}^{R} y_{I}$ and $y_{I} \chi_{I}^{R} x_{I}$. Then three cases are possible:

(1) there is $a_{-I}, b_{-I}$ such that $\left(y_{I}, a_{-I}\right) \in C^{k} \cap C^{k-1},\left(x_{I}, a_{-I}\right) \notin C_{\geq}^{k}$ and $\left(x_{I}, b_{-I}\right) \in C^{l} \cap C^{l-1},\left(y_{I}, b_{-I}\right) \notin C_{\geq}^{l}$. This clearly contradicts $\nu$-LinearityF.

(2) there is $a_{-I}, b_{-I}$ such that $\left(y_{I}, a_{-I}\right) \in I^{k},\left(x_{I}, a_{-I}\right) \notin I_{\geq}^{k}$ and $\left(x_{I}, b_{-I}\right) \in$ $I^{l},\left(y_{I}, b_{-I}\right) \notin I_{\geq}^{l}$. This clearly contradicts $\nu$-Linearity-I. 
(3) there is $a_{-I}, b_{-I}$ such that $\left(y_{I}, a_{-I}\right) \in C^{k} \cap C^{k-1},\left(x_{I}, a_{-I}\right) \notin C_{>}^{k}$ and $\left(x_{I}, b_{-I}\right) \in I^{l},\left(y_{I}, b_{-I}\right) \notin I_{\geq}^{l}$. Suppose

- $k \leq l$. By Lemma 1, there is then $c_{-I}:\left(x_{I}, c_{-I}\right) \in C^{l} \cap C^{l-1}$. Let us now consider $\left(y_{I}, c_{-I}\right)$. Three cases are possible. (a) $\left(y_{I}, c_{-I}\right) \in C^{l} \cap C^{l-1}$. Then, by $\nu$-Thinness and because $\left(y_{I}, a_{-I}\right) \in C^{k} \cap C^{k-1}$, we find that $\left(x_{I}, a_{-I}\right) \in C^{k} \cap C^{k-1}$, which is false. So, $\left(y_{I}, c_{-I}\right) \notin C^{l} \cap C^{l-1}$. (b) $\left(y_{I}, c_{-I}\right) \in I_{>}^{l}$. Then, by $\nu$-Linearity-I, $\left(x_{I}, b_{-I}\right) \in I^{l},\left(y_{I}, c_{-I}\right) \in I_{>}^{l}$ and $\left(x_{I}, c_{-I}\right) \in C^{l} \cap C^{l-1}$ yield $\left(y_{I}, b_{-I}\right) \in I_{\geq}^{l}$. A contradiction. (c) $\left(y_{I}, c_{-I}\right) \notin$ $C_{>}^{l}$. Then, by $\nu$-Linearity-F $,\left(x_{I}, c_{-I}\right) \in C^{l} \cap C^{l-1},\left(y_{I}, a_{-I}\right) \in C^{k} \cap C^{k-1}$ and $\left(y_{I}, c_{-I}\right) \notin C_{\geq}^{l}$ yield $\left(x_{I}, a_{-I}\right) \in C_{\geq}^{k}$. A contradiction.

- $k>l$. Since $\left(y_{I}, a_{-I}\right) \in C^{k} \cap C^{k-1}$, the sets $X_{i}$ being unbounded, there is $c_{-I}:\left(y_{I}, c_{-I}\right) \in I_{\geq}^{k}$. Using $\nu$-Linearity-I and the fact that $\left(y_{I}, c_{-I}\right) \in$ $I_{\geq}^{k},\left(x_{I}, b_{-I}\right) \in I^{l}$ and $\left(y_{I}, b_{-I}\right) \notin I_{\geq}^{l}$, we obtain $\left(x_{I}, c_{-I}\right) \in I_{\geq}^{k}$. By Lemma 1 , there is then $d_{-I}:\left(x_{I}, d_{-I}\right) \in C^{k} \cap C^{k-1}$. By $\nu$-Linearity-F and the fact that $\left(x_{I}, d_{-I}\right) \in C^{k} \cap C^{k-1},\left(y_{I}, a_{-I}\right) \in C^{k} \cap C^{k-1}$ and $\left(x_{I}, a_{-I}\right) \notin C_{\geq}^{k}$, we find $\left(y_{I}, d_{-I}\right) \in C_{\geq}^{k}$. By $\nu$-Thinness, we cannot have $\left(y_{I}, d_{-I}\right) \in C^{\bar{k}} \cap C^{k-1}$. So, $\left(y_{I}, d_{-I}\right) \in I_{>}^{k}$. Finally, using $\nu$-Linearity-I and the fact that $\left(y_{I}, d_{-I}\right) \in I_{\geq}^{k},\left(x_{I}, b_{-I}\right) \in I^{l}$ and $\left(y_{I}, b_{-I}\right) \notin I_{\geq}^{l}$, we find $\left(x_{I}, d_{-I}\right) \in I_{\geq}^{k}$. A contradiction.

We will later construct mappings $u_{i}$ such that $u_{i}\left(x_{i}\right) \geq u_{i}\left(y_{i}\right)$ iff $x_{i} \succsim_{i}^{R} y_{i}$. Notice that, because $\succsim_{i}^{R}$ is a weak order, the interval topology is the same as the order topology. Hence $X_{i}$ is connected in the order topology and we will always use the order topology in the sequel.

Lemma 3 The structure $\left\langle X,\left(C^{k}\right)_{k \in R}\right\rangle$ is monotonic with respect to $\succsim_{I}^{R}$, i.e. for all $x, y \in X$ and all $I \in N$,

- $y \in I^{k}$ and $x_{I} \succsim_{I}^{R} y_{I}$ implies $\left(x_{I}, y_{-I}\right) \in I_{\geq}^{k}$.

- $y \in C^{k} \cap C^{k-1}$ and $x_{I} \succsim_{I}^{R} y_{I}$ implies $\left(x_{I}, y_{-I}\right) \in C_{\geq}^{k}$.

- $y \in I^{k}$ and $y_{I} \succsim_{I}^{R} x_{I}$ implies $\left(x_{I}, y_{-I}\right) \notin C_{\geq}^{k+1}$.

- $y \in C^{k} \cap C^{k-1}$ and $y_{I} \succsim_{I}^{R} x_{I}$ implies $\left(x_{I}, y_{-I}\right) \in C_{<}^{k}$.

- $y \in C^{k}$ and $x_{I} \sim_{I}^{R} y_{I}$ implies $\left(x_{I}, y_{-I}\right) \in C^{k}$.

Proof. Monotonicity easily follows from the definition of $\succsim_{I}^{R}$.

Lemma 4 If the structure $\left\langle X,\left(C^{k}\right)_{k \in R}\right\rangle$ satisfies $\nu$-Thinness, then it is strictly monotonic with respect to any relation $\succsim_{I}^{R}$ with $\# I=\nu$, i.e. for all $x, y \in X$ and all $I \in N$,

(1) $y \in C^{k} \cap C^{k-1}$ and $x_{I} \succ_{I}^{R} y_{I}$ implies $\left(x_{I}, y_{-I}\right) \in I_{>}^{k}$.

(2) $y \in C^{k} \cap C^{k-1}$ and $y_{I} \succ_{I}^{R} x_{I}$ implies $\left(x_{I}, y_{-I}\right) \notin C_{\geq}^{k}$. 
Proof. We prove (1). Suppose that $y \in C^{k} \cap C^{k-1}$ and $x_{I} \succ_{I}^{R} y_{I}$. By monotonicity (see Lemma 3$),\left(x_{I}, y_{-I}\right) \in C_{\geq}^{k}$. If $\left(x_{I}, y_{-I}\right) \in C^{k} \cap C^{k-1}$, then $y \in C^{k} \cap C^{k-1}$ and $\nu$-Thinness imply that $x_{I} \sim_{I}^{R} y_{I}$. This is a contradiction. So, $\left(x_{I}, y_{-I}\right) \in I_{\geq}^{k}$. The proof of $(2)$ is similar.

Lemma 5 Under the conditions of Prop. 1, $\left\langle X,\left(C^{1}, C^{2}\right)\right\rangle$ satisfies 2-LinearityF.

Proof. Suppose, contrary to 2-Linearity-F,

$$
\begin{aligned}
& \left(x_{i}, x_{j}, a_{-i j}\right) \in C^{1} \cap C^{2}, \quad\left(y_{i}, y_{j}, a_{-i j}\right) \in I^{1}, \\
& \left(y_{i}, y_{j}, b_{-i j}\right) \in C^{1} \cap C^{2}, \quad\left(x_{i}, x_{j}, b_{-i j}\right) \in I^{1} .
\end{aligned}
$$

There are 4 possible cases.

- $y_{i} \succsim_{i}^{R} x_{i}, y_{j} \succsim_{j}^{R} x_{j}$. This contradicts monotonicity (see Lemma 3).

- $x_{i} \succsim_{i}^{R} y_{i}, x_{j} \succsim_{j}^{R} y_{j}$. This also contradicts monotonicity.

- $x_{i} \succ_{i}^{R} y_{i}, y_{j} \succ_{j}^{R} x_{j}$. By strict monotonicity (see Lemma 4), $\left(x_{i}, y_{j}, a_{-i j}\right) \in I^{2}$ and $\left(x_{i}, y_{j}, b_{-i j}\right) \in I^{2}$. By Restricted Solvability, there is $w_{j}$ s.t. $y_{j} \succ_{j}^{R} w_{j} \succ_{j}^{R}$ $x_{j}$ and $\left(x_{i}, w_{j}, b_{-i j}\right) \in C^{1} \cap C^{2}$. Because $w_{j} \succ_{j}^{R} x_{j}$, we find $\left(x_{i}, w_{j}, a_{-i j}\right) \in I^{2}$. Because $X_{j}$ is unbounded, there is $z_{j} \succ_{j}^{R} y_{j}$ and for each such $z_{j}$, by strict monotonicity, $\left(y_{i}, z_{j}, b_{-i j}\right) \in I^{2}$. Now, using 2-Linearity-I, $\left(y_{i}, z_{j}, b_{-i j}\right) \in I^{2}$, $\left(x_{i}, w_{j}, a_{-i j}\right) \in I^{2}$ and $\left(x_{i}, w_{j}, b_{-i j}\right) \in C^{1} \cap C^{2}$ yield $\left(y_{i}, z_{j}, a_{-i j}\right) \in I^{2}$. So, for any $z_{j} \succ_{j}^{R} y_{j},\left(y_{i}, z_{j}, a_{-i j}\right) \in I^{2}$. But, by Restricted Solvability, there is $z_{j}^{\prime} \succ_{j}^{R} y_{j}$ such that $\left(y_{i}, z_{j}^{\prime}, a_{-i j}\right) \in C^{1} \cap C^{2}$. A contradiction.

- $y_{i} \succ_{i}^{R} x_{i}, x_{j} \succ_{j}^{R} y_{j}$. Impossible for the same reasons as in the previous case.

Lemma 6 Under the conditions of Prop. 1, $\left\langle X,\left(C^{1}, C^{2}\right)\right\rangle$ satisfies 2-Thinness.

Proof. Suppose 2-Thinness does not hold. Then, for some $x_{i}, y_{i} \in X_{i}$, some $x_{j}, y_{j} \in X_{j}$ and some $a_{-i j}, b_{-i j} \in X_{-i j}$, we have
(a) $\left(x_{i}, x_{j}, a_{-i j}\right) \in C^{1} \cap C^{2}$,
(b) $\left(x_{i}, x_{j}, b_{-i j}\right) \in I^{2}$,
(c) $\left(y_{i}, y_{j}, a_{-i j}\right) \in C^{1} \cap C^{2}$,
(d) $\left(y_{i}, y_{j}, b_{-i j}\right) \in C^{1}$

or

$$
\begin{array}{cl}
\left(x_{i}, x_{j}, a_{-i j}\right) & \in C^{1} \cap C^{2}, \quad\left(x_{i}, x_{j}, b_{-i j}\right) \in C^{2}, \\
\left(y_{i}, y_{j}, a_{-i j}\right) & \in C^{1} \cap C^{2}, \quad\left(y_{i}, y_{j}, b_{-i j}\right) \in I^{1} .
\end{array}
$$

We will present the proof only for the first case. The second case is similar. The proof then proceeds in three steps. 
Step 1. By monotonicity, (b) and (d) imply that we do not have $\left[x_{i} \sim_{i}^{R} y_{i}\right.$ and $\left.x_{j} \sim_{j}^{R} y_{j}\right]$. Then, using monotonicity and strict monotonicity, (a) and (c) imply $\left[x_{i} \succ_{i}^{R} y_{i}\right.$ and $\left.y_{j} \succ_{j}^{R} x_{j}\right]$ or $\left[y_{i} \succ_{i}^{R} x_{i}\right.$ and $\left.x_{j} \succ_{j}^{R} y_{j}\right]$. For the sake of definiteness, and without loss of generality, we assume $x_{i} \succ_{i}^{R} y_{i}$ and $y_{j} \succ_{j}^{R} x_{j}$.

Step 2. Let us show that $\left(w_{i}, x_{j}, b_{-i j}\right) \in I^{2}$, for some $w_{i} \in X_{i}$ such that $x_{i} \succ_{i}^{R} w_{i}$. There are three cases.

(1) $\left(y_{i}, x_{j}, b_{-i j}\right) \in I^{2}$ and we are done.

(2) $\left(y_{i}, x_{j}, b_{-i j}\right) \in C^{1} \cap C^{2}$. Because $X_{i}$ is connected, there is $w_{i}: x_{i} \succ_{i}^{R}$ $w_{i} \succ_{i}^{R} y_{i}$. By strict monotonicity, $\left(w_{i}, x_{j}, b_{-i j}\right) \in I^{2}$.

(3) $\left(y_{i}, x_{j}, b_{-i j}\right) \in I^{1}$. Then, by Restricted Solvability, there is $w_{i}^{\prime}:\left(w_{i}^{\prime}, x_{j}, b_{-i j}\right) \in$ $C^{1} \cap C^{2}$. Of course, by strict monotonicity, $x_{i} \succ_{i}^{R} w_{i}^{\prime}$. And we are back to case $(2)$.

Notice that $\left(w_{i}, x_{j}, a_{-i j}\right) \in I^{1}$ by strict monotonicity.

Step 3. We now consider two cases.

(1) $\left(y_{i}, y_{j}, b_{-i j}\right) \in I^{1}$. We follow the same reasoning as in step 2 , and we find $z_{i}: z_{i} \succ_{i}^{R} y_{i}$ and $\left(z_{i}, y_{j}, b_{-i j}\right) \in I^{1}$. Because $\left(y_{i}, y_{j}, a_{-i j}\right) \in C^{1} \cap C^{2}, z_{i} \succ_{i}^{R}$ $y_{i}$ and by strict monotonicity, $\left(z_{i}, y_{j}, a_{-i j}\right) \in I^{2}$. Now, using 2-Linearity$\mathrm{I},\left(w_{i}, x_{j}, b_{-i j}\right) \in I^{2}$ and $\left(z_{i}, y_{j}, a_{-i j}\right) \in I^{2}$ yields $\left(z_{i}, y_{j}, b_{-i j}\right) \in I^{2}$ or $\left(w_{i}, x_{j}, a_{-i j}\right) \in I^{2}$. A contradiction.

(2) $\left(y_{i}, y_{j}, b_{-i j}\right) \in C^{1} \cap C^{2}$. Take any $l \in N \backslash\{i, j\}$. Because $X_{l}$ is connected and unbounded, we can follow the same reasoning as in step 2, and we find $z_{l}: b_{l} \succ_{l}^{R} z_{l}$ and $\left(x_{i}, x_{j}, z_{l}, b_{-i j l}\right) \in I^{2}$. By strict monotonicity, $\left(y_{i}, y_{j}, z_{l}, b_{-i j l}\right) \in I^{1}$ and we are back to case 1 .

So, 2-Thinness must hold.

Lemma 7 Under the conditions of Prop. 1, $\left\langle X,\left(C^{1}, C^{2}\right)\right\rangle$ satisfies $(n-1)$ Thinness and $(n-2)$-Thinness.

Proof. Suppose $\left(x_{I}, a_{-I}\right) \in C^{1} \cap C^{2}$ and $\left(y_{I}, a_{-I}\right) \in C^{1} \cap C^{2}$, with \#I=m and $m=n-1$ or $n-2$. Suppose $m$-Thinness does not hold. There is then $b_{-I}$ such that (a) $\left(x_{I}, b_{-I}\right) \in I^{2},\left(y_{I}, b_{-I}\right) \in I^{1}$ or $(\mathrm{b})\left(x_{I}, b_{-I}\right) \in I^{2},\left(y_{I}, b_{-I}\right) \in$ $C^{1} \cap C^{2}$ or $(\mathrm{c})\left(x_{I}, b_{-I}\right) \in I^{1},\left(y_{I}, b_{-I}\right) \in C^{1} \cap C^{2}$. Let us first consider case (a). By Lemma 1, there is $z_{I}:\left(z_{I}, b_{-I}\right) \in C^{1} \cap C^{2}$. If $\left(z_{I}, a_{-I}\right) \in C^{1} \cap C^{2}$ then $(n-m)$-Thinness is violated. If $\left(z_{I}, a_{-I}\right) \in I^{1}$, then $(n-m)$-Linearity-F is violated. If $\left(z_{I}, a_{-I}\right) \in I^{2}$, then $(n-m)$-Linearity-I is violated.

We now handle case (b). It violates $(n-m)$-Thinness. Finally, case (c) also violates $(n-m)$-Thinness. 
Lemma 8 Under the conditions of Prop. $1,\left(x_{I}, a_{-I}\right) \in C^{2}$ and $\left(y_{I}, a_{-I}\right) \in C^{1}$ imply $x_{I} \succsim_{I}^{R} y_{I}$, for all $I \subset N$ such that $\# I \leq 2$.

Proof. Let $m=\# I$. By Lemmas 5 and 6, 2-Linearity-F and 2-Thinness are satisfied. Hence, $\succsim_{I}^{R}$ is a weak order, monotonic and strictly monotonic (Lemmas 3 and 4). Suppose $\left(x_{I}, a_{-I}\right) \in C^{2}$ and $\left(y_{I}, a_{-I}\right) \in C^{1}$ but NOT $x_{I} \succsim_{I}^{R} y_{I}$. Because $\succsim_{I}^{R}$ is complete, we have $y_{I} \succ_{I}^{R} x_{I}$. If $\left(x_{I}, a_{-I}\right) \in I^{2}$, the monotonicity of $\succsim_{I}^{R}$ immediately implies $\left(y_{I}, a_{-I}\right) \in I^{2}$. A contradiction. If $\left(x_{I}, a_{-I}\right) \in C^{1} \cap C^{2}$, the strict monotonicity of $\succsim_{I}^{R}$ immediately implies $\left(y_{I}, a_{-I}\right) \in I^{2}$. A contradiction. So, we must have $x_{I} \succsim_{I}^{R} y_{I}$.

Lemma 9 Under the conditions of Prop. 1, $\left(x_{I}, a_{-I}\right) \in C^{2}$ and $\left(y_{I}, a_{-I}\right) \in I^{1}$ imply $x_{I} \succ_{I}^{R} y_{I}$, for all $I \subset N$ such that $\# I \leq 2$. Similarly, $\left(x_{I}, a_{-I}\right) \in I^{2}$ and $\left(y_{I}, a_{-I}\right) \in C^{1}$ imply $x_{I} \succ_{I}^{R} y_{I}$.

Proof. Using Lemma 8, the proof is straightforward.

\section{A.2 Proof of Proposition 1}

Before proving Theorem 1, we state a result of Chateauneuf and Wakker (1993) that will be central below (their Corollary 2.3).

\section{Theorem 4 [Chateauneuf and Wakker (1993)] Assume}

- $\left(Y_{i}\right)_{i \in M}$ are non-empty sets endowed with non-trivial weak orders $\geq_{i}^{\circ}$ and their order topologies; $M=\{1, \ldots, m\}, m \geq 2$;

- $\prod_{i \in M} Y_{i}$ is endowed with the product topology;

- A weak order $\geq^{\circ}$ is given on an open set $E \subset \prod_{i \in M} Y_{i}$; it is

- monotonic, i.e. $y_{i} \geq_{i}^{\circ} y_{i}^{\prime} \forall i \in M \Rightarrow y \geq^{\circ} y^{\prime}$;

- strictly monotonic, i.e. $y_{i} \geq_{i}^{\circ} y_{i}^{\prime} \forall i \in M$ and $y_{j}>_{j}^{\circ} y_{j}^{\prime}$ for some $j \in M \Rightarrow$ $y>^{\circ} y^{\prime}$, and

- continuous, i.e. for all $y \in E,\left\{y^{\prime} \in E: y^{\prime} \geq^{\circ} y\right\}$ and $\left\{y^{\prime} \in E: y \geq^{\circ} y^{\prime}\right\}$ are closed in $E$;

- The following sets are connected:

- The topological interior of $E$ denoted by $\operatorname{Int}(E)$;

- all sets of the form: $\left\{y \in \operatorname{Int}(E): y_{i}=s_{i}\right\}$ for some $i \in M$ and $s_{i} \in Y_{i}$;

- all equivalence classes of $\geq^{\circ}$ in $\operatorname{Int}(E)$;

Assume also one of the following:

- Independence (if $m \geq 3)$, i.e. $\left(y_{i}, a_{-i}\right) \geq^{\circ}\left(y_{i}, b_{-i}\right) \Leftrightarrow\left(y_{i}^{\prime}, a_{-i}\right) \geq^{\circ}\left(y_{i}^{\prime}, b_{-i}\right)$ for all $y_{i}, y_{i}^{\prime} \in Y_{i}$ and all $a_{-i}, b_{-i} \in Y_{-i}$; 
- Thomsen (if $m=2)$, i.e. $\left[\left(y_{i}, a_{-i}\right)={ }^{\circ}\left(y_{i}^{\prime}, b_{-i}\right)\right.$ and $\left.\left(y_{i}^{\prime}, c_{-i}\right)={ }^{\circ}\left(y_{i}^{\prime \prime}, a_{-i}\right)\right] \Rightarrow$ $\left(y_{i}, c_{-i}\right)=^{\circ}\left(y_{i}^{\prime \prime}, b_{-i}\right)$, for all $y_{i}, y_{i}^{\prime}, y_{i}^{\prime \prime} \in Y_{i}$ and all $a_{-i}, b_{-i}, c_{-i} \in Y_{-i}$.

There is then a continuous additive representation of $\geq^{\circ}$. It is unique up to $m$ positive affine transformations with the same multiplicative constant.

We are now ready to prove Proposition 1.

Proof of Proposition 1. Let $S=\left\{x_{-1} \in X_{-1}:\left(x_{1}, x_{-1}\right) \in C^{1} \cap\right.$ $C^{2}$ for some $\left.x_{1} \in X_{1}\right\}$. Note that $S$ is the projection of $C^{1} \cap C^{2}$ on $X_{-1}$. We now prove a series of statements about $S$.

$S$ is not empty.

Because $\left(C^{1}, C^{2}\right)$ is not degenerate, $\exists x, y: x \in I^{1}, y \in I^{2}$. We then just need to apply Lemma 1.

$S$ is open.

This we prove by showing that for any $x_{-1}$ in $S$, there is an open box containing $x_{-1}$. Because $x_{-1} \in S$, we know there is $x_{1} \in X_{1}:\left(x_{1}, x_{-1}\right) \in C^{1} \cap C^{2}$. Since $X_{1}$ is unbounded, there are $x_{1}^{\prime}, x_{1}^{\prime \prime}: x_{1}^{\prime} \succ_{1}^{R} x_{1} \succ_{1}^{R} x_{1}^{\prime \prime}$. By Lemma 4, $\left(x_{1}^{\prime}, x_{-1}\right) \in I^{2}$ and $\left(x_{1}^{\prime \prime}, x_{-1}\right) \in I^{1}$. Similarly, for any $i \in N \backslash\{1\}$, there is $x_{i}^{\prime}, x_{i}^{\prime \prime}: x_{i}^{\prime} \succ_{i}^{R} x_{i} \succ_{i}^{R} x_{i}^{\prime \prime}$ and $\left(x_{1}, x_{-1 i}, x_{i}^{\prime}\right) \in I^{2}$ and $\left(x_{1}, x_{-1 i}, x_{i}^{\prime \prime}\right) \in I^{1}$. Consider now the element $\left(x_{1}^{\prime}, x_{-1 i}, x_{i}^{\prime \prime}\right)$ (we will later consider $\left(x_{1}^{\prime \prime}, x_{-1 i}, x_{i}^{\prime}\right)$ ). Two cases (non-exclusive) can arise:

- $\left(x_{1}^{\prime}, x_{-1 i}, x_{i}^{\prime \prime}\right) \in C^{1}$. Then, by Restricted Solvability, there is $y_{i}:\left(x_{1}^{\prime}, x_{-1 i}, y_{i}\right) \in$ $C^{1} \cap C^{2}$. So, $\left(x_{-1 i}, y_{i}\right) \in S$.

- $\left(x_{1}^{\prime}, x_{-1 i}, x_{i}^{\prime \prime}\right) \in C^{2}$. Then, by Restricted Solvability, there is $y_{1}:\left(y_{1}, x_{-1 i}, x_{i}^{\prime \prime}\right) \in$ $C^{1} \cap C^{2}$. So, $\left(x_{-1 i}, x_{i}^{\prime \prime}\right) \in S$.

Let $z_{i}^{\prime \prime}$ denote $y_{i}$ in the first case and $x_{i}^{\prime \prime}$ in the second case. Remark that $x_{i} \succ_{i}^{R} z_{i}^{\prime \prime}$. Let us turn now to the element $\left(x_{1}^{\prime \prime}, x_{-1 i}, x_{i}^{\prime}\right)$. We can repeat our reasoning and we find $z_{i}^{\prime}$ with $z_{i}^{\prime} \succ_{i}^{R} x_{i}$ such that $\left(x_{-1 i}, z_{i}^{\prime}\right) \in S$. Let $] z_{i}^{\prime \prime}, z_{i}^{\prime}[i$ denote the set $\left\{z_{i} \in X_{i}: z_{i}^{\prime \prime} \prec_{i}^{R} z_{i} \prec_{i}^{R} z_{i}^{\prime}\right\}$. It is clear that, for any $z_{i}$ in $] z_{i}^{\prime \prime}, z_{i}^{\prime}[i$, $\left(x_{-1 i}, z_{i}\right) \in S$. Hence, we have found an open box

$$
\left.B\left(x_{-1}\right)=\prod_{i \in N \backslash\{1\}}\right] z_{i}^{\prime \prime}, z_{i}^{\prime}{ }_{i}
$$

containing $x_{-1}$. Note that $B\left(x_{-1}\right)$ is a connected subspace of $S$ for each interval $] z_{i}^{\prime \prime}, z_{i}^{\prime}[i$ is obviously connected.

For any $x_{-i j}$, the set $T\left(x_{-i j}\right)=\left\{\left(x_{i}, x_{j}, x_{-i j}\right) \in C^{1} \cap C^{2}\right\}$ is connected.

Assume that $T\left(x_{-i j}\right)$ is not empty (otherwise it is trivially connected). De- 
fine then $T_{i}\left(x_{-i j}\right)=\left\{x_{i}:\left(x_{i}, x_{j}, x_{-i j}\right) \in C^{1} \cap C^{2}\right.$ for some $\left.x_{j}\right\}$. By Restricted Solvability, this set is an interval of $X_{i}$ and, hence, connected. Let $T_{i}^{\prime}\left(x_{-i j}\right)$ be the quotient $T_{i}\left(x_{-i j}\right) / \sim_{i}^{R}$. Define the relation $\succsim^{T}$ on $T\left(x_{-i j}\right)$ by $x \succsim^{T} y \Leftrightarrow x_{i} \succsim_{i}^{R} y_{i}$. The relation $\succsim^{T}$ is clearly a weak order. Define $T^{\prime}\left(x_{-i j}\right)$ by $T^{\prime}\left(x_{-i j}\right)=T\left(x_{-i j}\right) / \sim^{T}$. Define the mapping $f: T_{i}^{\prime}\left(x_{-i j}\right) \rightarrow T^{\prime}\left(x_{-i j}\right)$ by $f\left(x_{i}\right)=x$ iff $\left(x_{i}, x_{j}, x_{-i j}\right) \in T^{\prime}\left(x_{-i j}\right)$ for some $x_{j}$. This mapping is order preserving and surjective (actually, it is bijective). So, $T_{i}^{\prime}\left(x_{-i j}\right)$ and $T^{\prime}\left(x_{-i j}\right)$ are homeomorphic (Munkres, 2000, Exercise 7.a, p. 158). Consequently, $T_{i}^{\prime}\left(x_{-i j}\right)$ being connected, $T^{\prime}\left(x_{-i j}\right)$ is also connected and so is $T\left(x_{-i j}\right)$.

For any $x_{i}$, the set $Q\left(x_{i}\right)=\left\{\left(x_{i}, x_{-i}\right) \in C^{1} \cap C^{2}\right\}$ is connected.

Before proving this, let us recall a standard result (hereafter called R1) in topology: the union of a collection of connected sets that have a point in common is connected (Munkres, 2000, Th. 23.3). Choose now a point $\left(x_{i}, x_{-i}^{*}\right) \in$ $Q\left(x_{i}\right)$. For any other point $\left(x_{i}, x_{-i}\right) \in Q\left(x_{i}\right)$, we can 'go' from $\left(x_{i}, x_{-i}^{*}\right)$ to $\left(x_{i}, x_{-i}\right)$ moving consecutively in a succession of at most $n-2$ sets of the form $T\left(x_{-j j^{\prime}}\right)$ with $x_{i}$ fixed. Let $P\left(x_{-i}\right)$ be the union of those $n-2$ sets. Remark that these sets all have a point in common with the next one in the succession. We can therefore apply R1 $n-3$ times and we find that $P\left(x_{-i}\right)$ is connected. Remark now that

$$
Q\left(x_{i}\right)=\bigcup_{\left(x_{i}, x_{-i}\right) \in Q\left(x_{i}\right)} P\left(x_{-i}\right) .
$$

It is an arbitrary union of connected sets with the point $\left(x_{i}, x_{-i}^{*}\right)$ in common. Hence, according to R1, it is connected.

$C^{1} \cap C^{2}$ is connected.

Choose a point $x^{*} \in C^{1} \cap C^{2}$. For any other point $x \in C^{1} \cap C^{2}$, we can 'go' from $x^{*}$ to $x$ moving first in a set of the form $Q\left(x_{1}^{*}\right)$ to a point having at least one coordinate $i$ in common with $x$ and then in a set of the form $Q\left(x_{i}\right)$. Let $P^{\prime}(x)$ be the union of these two sets. Of course, $P^{\prime}(x)$ is connected. Remark now that

$$
C^{1} \cap C^{2}=\bigcup_{x \in C^{1} \cap C^{2}} P^{\prime}(x) .
$$

It is an arbitrary union of connected sets all having the point $x^{*}$ in common. Hence, thanks again to R1, it is connected.

The set $S$ is connected.

First note that $S$ is the projection of the connected set $C^{1} \cap C^{2}$ on $X_{-1}$. A projection mapping is always continuous since the image of an open set under the inverse of a projection mapping is open, by construction (Munkres, 2000, p. 87). The image of a connected set under a continuous mapping is connected 
(Munkres, 2000, Th. 23.5). Hence $S$ is connected.

For any $x_{i}$, with $i \neq 1$, the set $\left\{y_{-1} \in S: y_{i}=x_{i}\right\}$ is connected.

This is obvious since this set is the projection of the connected set $Q\left(x_{i}\right)$ on $X_{-1}$.

Let us define $\succsim^{*}$ on $S$ by letting

$$
x_{-1} \succsim^{*} y_{-1} \Leftrightarrow \exists x_{1} \in X_{1}:\left\{\begin{array}{l}
\left(x_{1}, x_{-1}\right) \in C^{2} \\
\text { and } \\
\left(x_{1}, y_{-1}\right) \in C^{1} .
\end{array}\right.
$$

We will show that this relation ${ }^{4}$ admits an additive representation but, because it is not defined on the complete product set $X_{-1}$ but only on a subset of it, we cannot use the standard representation theorems of, for example, Debreu (1960) or Krantz et al. (1971) but we must use special theorems dealing with incomplete product sets. Before doing so, we prove a series of statements about ${ }^{*}$.

$\succsim^{*}$ is complete.

Suppose $\succsim^{*}$ is not complete. Then, $\nexists x_{1} \in X_{1}:\left(x_{1}, x_{-1}\right) \in C^{2},\left(x_{1}, y_{-1}\right) \in C^{1}$ and $\nexists x_{1}^{\prime} \in X_{1}:\left(x_{1}^{\prime}, x_{-1}\right) \in C^{1},\left(x_{1}^{\prime}, y_{-1}\right) \in C^{2}$. So, for all $y_{1} \in X_{1},\left(y_{1}, x_{-1}\right)$ and $\left(y_{1}, y_{-1}\right)$ both belong to $I^{1}$ or to $I^{2}$. We know this is false since, for at least one $z_{1} \in X_{1},\left(z_{1}, x_{-1}\right) \in C^{1} \cap C^{2}$.

$\succsim^{*}$ is transitive.

Suppose $x_{-1} \succsim^{*} y_{-1}$ and $y_{-1} \succsim^{*} z_{-1}$. So, there is $x_{1}:\left(x_{1}, x_{-1}\right) \in C^{2},\left(x_{1}, y_{-1}\right) \in$ $C^{1}$ and $y_{1}:\left(y_{1}, y_{-1}\right) \in C^{2},\left(y_{1}, z_{-1}\right) \in C^{1}$. Then two cases are possible: (1) $\left(y_{1}, y_{-1}\right) \in C^{2} \cap C^{1}$. By 1-Thinness, $\left(x_{1}, z_{-1}\right) \in C^{1}$. (2) $\left(y_{1}, y_{-1}\right) \in I^{2}$. By 1-Linearity-I, $\left(x_{1}, z_{-1}\right) \in C^{1}$.So, $x_{-1} \succsim^{*} z_{-1}$.

$\succsim^{*}$ is monotonic (in the sense of Chateauneuf and Wakker, 1993).

Suppose $x_{-1}, y_{-1} \in S$ and $y_{i} \succsim_{i}^{R} x_{i}, \forall i \in N \backslash\{1\}$. Since $x_{-1} \in S$, there is $a_{1}:\left(x_{-1}, a_{1}\right) \in C^{1} \cap C^{2}$. Applying Lemma $3(n-1)$ times, we successively find $\left(x_{-12}, y_{2}, a_{1}\right) \in C^{2},\left(x_{-123}, y_{23}, a_{1}\right) \in C^{2}, \ldots,\left(y_{-1}, a_{1}\right) \in C^{2}$. This combined with $\left(x_{-1}, a_{1}\right) \in C^{1} \cap C^{2}$ implies $y_{-1} \succsim^{*} x_{1}$.

$\overline{4 \text { It can }}$ be shown that $\succsim^{*}$ is identical to the restriction of $\succsim_{-1}^{R}$ to $S$. 
$\succsim^{*}$ is strictly monotonic (in the sense of Chateauneuf and Wakker, 1993).

Suppose $x_{-1}, y_{-1} \in S, y_{i} \succsim_{i}^{R} x_{i}, \forall i \in N \backslash\{1\}$ and $y_{j} \succ_{j}^{R} x_{j}$, for some $j \in N \backslash\{1\}$. Since $x_{-1} \in S$, there is $a_{1}:\left(x_{-1}, a_{1}\right) \in C^{1} \cap C^{2}$. Applying Lemma 4 , we find $\left(x_{-1 j}, y_{j}, a_{1}\right) \in I^{2}$. Applying then Lemma $3(n-2)$ times, we successively find $\left(x_{-1 j 2}, y_{j 2}, a_{1}\right) \in I^{2},\left(x_{-1 j 23}, y_{j 23}, a_{1}\right) \in I^{2}, \ldots,\left(y_{-1}, a_{1}\right) \in I^{2}$. This combined with $\left(x_{-1}, a_{1}\right) \in C^{1} \cap C^{2}$ implies $y_{-1} \succsim^{*} x_{1}$.

$\succsim^{*}$ is continuous.

For any $x_{-1} \in S$, let $R^{+}\left(x_{-1}\right)=\left\{y_{-1} \in S: y_{-1} \succ^{*} x_{-1}\right\}$. By definition of $S$, there is $x_{1}^{*}$ such that $\left(x_{1}^{*}, x_{-1}\right) \in C^{1} \cap C^{2}$. For any $y_{-1} \in Q\left(x_{1}^{*}\right), y_{-1} \sim^{*} x_{-1}$. Similarly, for any $y_{-1} \in Q\left(x_{1}^{*}\right)$ and $z_{-1} \in R^{+}\left(x_{-1}\right)$, we have $z_{-1} \succ^{*} y_{-1}$. We now prove that $R^{+}\left(x_{-1}\right)$ is open. This we do by showing that for any $z_{-1}$ in $R^{+}\left(x_{-1}\right)$ there is an open box in $R^{+}\left(x_{-1}\right)$ containing $z_{-1}$. Because $z_{-1}$ lies in $S$, we can construct an open box $B\left(z_{-1}\right)$ in $S$ around $z_{-1}$ as in (A.1).

$$
\left.B\left(z_{-1}\right)=\prod_{i \in N \backslash\{1\}}\right] z_{i}^{\prime \prime}, z_{i}^{\prime}[i
$$

If this box lies entirely within $R^{+}\left(x_{-1}\right)$ then we are done. If not, then take any $y_{-1} \in Q\left(x_{1}^{*}\right)$ such that $z_{i} \succ_{i}^{R} y_{i}, \forall i \in N \backslash\{1\}$. Because $X_{i}$ is connected, there are $w_{i}: z_{i} \succ_{i}^{R} w_{i} \succ_{i}^{R} y_{i}, \forall i \in N \backslash\{1\}$. By strict monotonicity, $w_{-1} \succ^{*} y_{-1}$ and, so, $w_{-1} \in R^{+}\left(x_{-1}\right)$. Consider then the box

$$
\left.\prod_{i \in N \backslash\{1\}}\right] w_{i}, z_{i}^{\prime}{ }_{i}
$$

It is open and lies entirely within $R^{+}\left(x_{-1}\right)$. Hence, $R^{+}\left(x_{-1}\right)$ is open. Let us now define $R^{-}\left(x_{-1}\right)=\left\{y_{-1} \in S: x_{-1} \succ^{*} y_{-1}\right\}$. Following the same reasoning as above, we can show that $R^{-}\left(x_{-1}\right)$ is open. So, we have proven that ${ }^{*}$ is continuous.

$\succsim^{*}$ is Thomsen (in the sense of Chateauneuf and Wakker, 1993).

We need to check this only if $n=3$. Suppose $\succsim^{*}$ is not Thomsen. There exists then $x_{1}, y_{1}, z_{1} \in X_{1}, x_{2}, y_{2}, z_{2} \in X_{2}, x_{3}, y_{3}, z_{3} \in X_{3}$ such that $\left(x_{1}, y_{2}, z_{3}\right) \in$ $C^{1} \cap C^{2},\left(y_{1}, x_{2}, z_{3}\right) \in C^{1} \cap C^{2},\left(y_{1}, z_{2}, x_{3}\right) \in C^{1} \cap C^{2},\left(z_{1}, y_{2}, x_{3}\right) \in C^{1} \cap C^{2}$, $\left(x_{1}, z_{2}, y_{3}\right) \in C^{1} \cap C^{2}$ and $\left(z_{1}, x_{2}, y_{3}\right) \notin C^{1} \cap C^{2}$. If two of $x_{1}, y_{1}, z_{1}$ are equivalent in $\succsim_{1}^{R}$, it is easy to see that we have a contradiction. So, we can suppose without loss of generality that $z_{1} \succ_{1}^{R} y_{1} \succ_{1}^{R} x_{1}$. Consider first the case where $\left(z_{1}, x_{2}, y_{3}\right) \in I^{2}$. Then, $\left(x_{1}, y_{2}, z_{3}\right) \in C^{1} \cap C^{2}$ implies $\left(y_{1}, y_{2}, z_{3}\right) \in I^{2}$ and this implies in turn $y_{2} \succ_{2}^{R} x_{2}$. Similarly, $\left(y_{1}, z_{2}, x_{3}\right) \in C^{1} \cap C^{2}$ implies $\left(z_{1}, z_{2}, x_{3}\right) \in$ $I^{2}$ and this implies in turn $z_{2} \succ_{2}^{R} y_{2}$. Now, $z_{2} \succ_{2}^{R} y_{2}$ and $\left(x_{1}, y_{2}, z_{3}\right) \in C^{1} \cap C^{2}$ imply $\left(x_{1}, z_{2}, z_{3}\right) \in I^{2}$. This, combined with $\left(x_{1}, z_{2}, y_{3}\right) \in C^{1} \cap C^{2}$ implies $z_{3} \succ_{3}^{R} y_{3}$. Similarly, $y_{1} \succ_{1}^{R} x_{1}$ and $\left(y_{1}, z_{2}, x_{3}\right) \in C^{1} \cap C^{2}$ imply $\left(x_{1}, z_{2}, x_{3}\right) \in I^{1}$. 
From this, we find $y_{3} \succ_{3}^{R} x_{3}$. Because $X_{1}, X_{2}$ and $X_{3}$ are connected, there is $\left(w_{1}, w_{2}, w_{3}\right) \in I^{2}$ with $z_{1} \succ_{1}^{R} w_{1}, x_{2} \succ_{2}^{R} w_{2}$ and $y_{3} \succ_{3}^{R} w_{3}$. Because $y_{3} \succ_{3}^{R} w_{3}$, we find $\left(x_{1}, z_{2}, w_{3}\right) \in I^{1}$. Because $x_{2} \succ_{2}^{R} w_{2}$, we find $\left(y_{1}, w_{2}, z_{3}\right) \in I^{1}$. Because $z_{1} \succ_{1}^{R} w_{1}$, we find $\left(w_{1}, y_{2}, x_{3}\right) \in I^{1}$. By Connectedness, there is $w_{2}^{\prime} \succ_{2}^{R} z_{2}$ such that $\left(x_{1}, w_{2}^{\prime}, w_{3}\right) \in I^{1}$. By strict monotonicity, $\left(y_{1}, w_{2}^{\prime}, x_{3}\right) \in I^{2}$. By Connectedness, there is $w_{3}^{\prime} \succ_{3}^{R} z_{3}$ such that $\left(y_{1}, w_{2}, w_{3}^{\prime}\right) \in I^{1}$. By strict monotonicity, $\left(x_{1}, y_{2}, w_{3}^{\prime}\right) \in I^{2}$.

We now apply Double Cancellation with $\left(w_{1}, w_{2}, w_{3}\right),\left(y_{1}, w_{2}^{\prime}, x_{3}\right)$ and $\left(x_{1}, y_{2}, w_{3}^{\prime}\right)$. It yields that one of $\left(x_{1}, w_{2}^{\prime}, w_{3}\right),\left(y_{1}, w_{2}, w_{3}^{\prime}\right)$ and $\left(w_{1}, y_{2}, x_{3}\right)$ belongs to $C^{2}$. A contradiction.

The case where $\left(z_{1}, x_{2}, y_{3}\right) \in I^{1}$ is treated in a similar way.

$\succsim^{*}$ is Independent in the sense of Chateauneuf and Wakker (1993), that is $\left(x_{i}, a_{-i 1}\right) \succsim^{*}\left(x_{i}, b_{-i 1}\right)$ for some $x_{i}, i \neq 1$, and $a_{-i 1}, b_{-i 1} \in X_{-i 1}$ is equivalent to $\left(y_{i}, a_{-i 1}\right) \succsim^{*}\left(y_{i}, b_{-i 1}\right)$ for all $y_{i} \in X_{i}$.

Indeed, contrary to Independence of $\succsim^{*}$, suppose $\left(x_{i}, a_{-i 1}\right) \succsim^{*}\left(x_{i}, b_{-i 1}\right)$ and $\left(y_{i}, b_{-i 1}\right) \succ^{*}\left(y_{i}, a_{-i 1}\right)$. So, by definition of $\succsim^{*}$, there is $z_{1}, r_{1}:\left(z_{1}, x_{i}, a_{-i 1}\right) \in$ $C^{2},\left(z_{1}, x_{i}, b_{-i 1}\right) \in C^{1},\left(r_{1}, y_{i}, b_{-i 1}\right) \in C^{2},\left(r_{1}, y_{i}, a_{-i 1}\right) \in C^{1}$ and,

$$
\forall w_{1},\left(w_{1}, y_{i}, a_{-i 1}\right) \in C^{2} \Rightarrow\left(w_{1}, y_{i}, b_{-i 1}\right) \in I^{2} .
$$

By Lemma 8, $\left(z_{1}, x_{i}\right) \sim_{1 i}^{R}\left(r_{1}, y_{i}\right)$. So, by Lemma $3,\left(z_{1}, x_{i}, a_{-i 1}\right) \in C^{1} \cap C^{2}$, $\left(z_{1}, x_{i}, b_{-i 1}\right) \in C^{1} \cap C^{2},\left(r_{1}, y_{i}, b_{-i 1}\right) \in C^{1} \cap C^{2}$ and $\left(r_{1}, y_{i}, a_{-i 1}\right) \in C^{1} \cap C^{2}$. By (A.3), $\left(r_{1}, y_{i}, b_{-i 1}\right) \in I^{2}$. A contradiction.

The equivalence classes of $*^{*}$ are connected.

This is obvious since an equivalence class of $\succsim^{*}$ is the projection of a connected set $Q\left(x_{1}\right)$ on $X_{-1}$.

The statements we proved about $S$ and $\succsim^{*}$ imply the conditions of Theorem 4 (Corollary 2.3 of Chateauneuf and Wakker, 1993). To see this, substitute $X_{-1}$ by $Y, N \backslash\{1\}$ by $M, S$ by $E, \succsim^{*}$ by $\geq^{\circ}$ and $\succsim_{i}^{R}$ by $\geq^{\circ}$.

Hence, there exists $n-1$ mappings $v_{i}: X_{i} \rightarrow \mathbb{R}, i \in\{2, \ldots, n\}$, such that

$$
x_{-1} \succsim^{*} y_{-1} \Leftrightarrow \sum_{i=2}^{n} v_{i}\left(x_{i}\right) \geq \sum_{i=2}^{n} v_{i}\left(y_{i}\right) .
$$

Furthermore, the mappings $v_{i}$ are unique up to $n-1$ positive affine transformations with the same multiplicative constant. 
Define now $v_{1}: X_{1} \mapsto \mathbb{R}$ by letting

$$
v_{1}\left(x_{1}\right)=s^{2}-\sum_{i=2}^{n} v_{i}\left(x_{i}\right) \text { if }\left(x_{1}, x_{-1}\right) \in C^{1} \cap C^{2}
$$

where $s^{2}$ is any real number. The mapping $v_{1}$ is defined for all $x_{1}$ in $X_{1}$. Indeed, suppose there are $y_{1}, z_{1}$ such that $\left(y_{1}, x_{-1}\right),\left(z_{1}, x_{-1}\right) \in I^{1}$ for all $x_{-1} \in X_{-1}$. We necessarily have $y_{1} \sim_{1}^{R} z_{1}$ and there is no $w_{1} \in X_{1}$ such that $y_{1} \succ_{1}^{R} w_{1}$. Hence, $\left\langle X_{1}, \succsim_{1}^{R}\right\rangle$ has a minimal element, a contradiction.

The mapping $v_{1}$ is well-defined: if $\left(x_{1}, x_{-1}\right) \in C^{1} \cap C^{2}$ and $\left(x_{1}, y_{-1}\right) \in C^{1} \cap C^{2}$, then $x_{-1} \sim^{*} y_{-1}$ and, so, $\sum_{i=2}^{n} v_{i}\left(x_{i}\right)=\sum_{i=2}^{n} v_{i}\left(y_{i}\right)$.

It is easy to see that

$$
\left(x_{1}, x_{-1}\right) \in C^{1} \cap C^{2} \Rightarrow \sum_{i=1}^{n} v_{i}\left(x_{i}\right)=s^{2} .
$$

Suppose now that $\sum_{i=1}^{n} v_{i}\left(x_{i}\right)=s^{2}$ and $x \notin C^{1} \cap C^{2}$. There is then $y_{-1}$ such that $\left(x_{1}, y_{-1}\right) \in C^{1} \cap C^{2}$ and such that $\sum_{i=2}^{n} v_{i}\left(x_{i}\right)=\sum_{i=2}^{n} v_{i}\left(y_{i}\right)$. Hence, by the representation (A.4), $y_{-1} \sim^{*} x_{-1}$. So, according to the definition of $\succsim^{*}$,

$$
\begin{aligned}
& \exists w_{1} \in X_{1}:\left(w_{1}, x_{-1}\right) \in C^{2} \text { and }\left(w_{1}, y_{-1}\right) \in C^{1} \\
& \text { and } \\
& \exists z_{1} \in X_{1}:\left(z_{1}, x_{-1}\right) \in C^{1} \text { and }\left(z_{1}, y_{-1}\right) \in C^{2} .
\end{aligned}
$$

By Lemma 8, this amounts to $w_{1} \sim_{1}^{R} z_{1}$. Then, by Lemma $3,\left(w_{1}, x_{-1}\right) \in C^{1} \cap$ $C^{2}$ and $\left(w_{1}, y_{-1}\right) \in C^{1} \cap C^{2}$. By $(n-1)$-Thinness (Lemma 7$),\left(x_{1}, y_{-1}\right) \in C^{1} \cap C^{2}$ implies $\left(x_{1}, x_{-1}\right) \in C^{1} \cap C^{2}$. A contradiction. Hence,

$$
\sum_{i=1}^{n} v_{i}\left(x_{i}\right)=s^{2} \Leftrightarrow x \in C^{1} \cap C^{2}
$$

and, this is obvious,

$$
\begin{aligned}
& \sum_{i=1}^{n} v_{i}\left(x_{i}\right) \geq s^{2} \Leftrightarrow x \in C^{2} \\
& \sum_{i=1}^{n} v_{i}\left(x_{i}\right) \leq s^{2} \Leftrightarrow x \in C^{1},
\end{aligned}
$$

which is the additive representation we were looking for. Any positive affine transformation (the same one for all attributes) of the mappings $u_{i}$ also represents $\left\langle X,\left(C^{1}, C^{2}\right)\right\rangle$ provided that the threshold $s^{2}$ is adapted accordingly. 
We finally prove that $v_{1}$ is continuous. Consider any $x \in C^{1} \cap C^{2}$. Using the same reasoning as when we proved that $S$ is open, we find $x_{1}^{\prime}, x_{1}^{\prime \prime}, x_{2}^{\prime}$ and $x_{2}^{\prime \prime}$ such that $x_{1}^{\prime} \prec_{1}^{R} x_{1} \prec_{1}^{R} x_{1}^{\prime \prime},\left(x_{1}^{\prime}, x_{2}^{\prime}, x_{-12}\right) \in C^{1} \cap C^{2}$ and $\left(x_{1}^{\prime \prime}, x_{2}^{\prime \prime}, x_{-12}\right) \in C^{1} \cap C^{2}$. For each $y_{1}: x_{1}^{\prime} \precsim_{1}^{R} y_{1} \precsim_{1}^{R} x_{1}^{\prime \prime}$, there is $y_{2}:\left(y_{1}, y_{2}, x_{-12}\right) \in C^{1} \cap C^{2}$. Similarly, for each $y_{2}: x_{2}^{\prime \prime} \precsim_{2}^{R} y_{2} \precsim_{2}^{R} x_{2}^{\prime}$, there is $y_{1}:\left(y_{1}, y_{2}, x_{-12}\right) \in C^{1} \cap C^{2}$. Clearly, for any $y_{1} \prec_{1}^{R} y_{1}^{\prime}$, if $y_{2}$ and $y_{2}^{\prime}$ are such that $\left(y_{1}, y_{2}, x_{-12}\right) \in C^{1} \cap C^{2}$ and $\left(y_{1}^{\prime}, y_{2}^{\prime}, x_{-12}\right) \in C^{1} \cap C^{2}$, we have $y_{2} \succ_{2}^{R} y_{2}^{\prime}$. Hence, $\left[x_{1}^{\prime}, x_{1}^{\prime \prime}\right]_{1}$ and $\left[x_{2}^{\prime \prime}, x_{2}^{\prime}\right]_{2}$ being connected, the mapping $\psi:\left[x_{1}^{\prime}, x_{1}^{\prime \prime}\right]_{1} \rightarrow\left[x_{2}^{\prime \prime}, x_{2}^{\prime}\right]_{2}$ defined by $\psi\left(y_{1}\right)=y_{2}$ such that $\left(y_{1}, y_{2}, x_{-12}\right) \in C^{1} \cap C^{2}$ is continuous as well as $\psi^{-1}$. So, given $x \in C^{1} \cap C^{2}$, we can rewrite (A.5) as

$$
\begin{aligned}
v_{1}\left(y_{1}\right) & =s^{2}-\sum_{i=3}^{n} v_{i}\left(x_{i}\right)-v_{2}\left(x_{2}\right) \text { if }\left(y_{1}, x_{-1}\right) \in C^{1} \cap C^{2} \\
& =K-v_{2}\left(\psi\left(y_{1}\right)\right)
\end{aligned}
$$

for all $y_{1} \in\left[x_{1}^{\prime}, x_{1}^{\prime \prime}\right]_{1}$. Because $\psi$ and $v_{2}$ are continuous, $v_{1}$ is also continuous on $] x_{1}^{\prime}, x_{1}^{\prime \prime}\left[_{1}\right.$. Since this holds for all $x_{1}$ in $X_{1}, v_{1}$ is everywhere continuous.

\section{A.3 Proof of Theorem 1}

Before proving Theorem 1, we prove one lemma. Let $b_{i}$ and $t_{i}$ respectively denote the minimal and maximal elements (when they exist) of the quotient $X_{i}^{\prime}=X_{i} / \sim_{i}^{R}$. If $b_{j}$ exists for all $j \in N \backslash\{i\}$, define $b_{-i}=\left(b_{j}\right)_{j \in N \backslash\{i\}}$. If $t_{j}$ exists for all $j \in N \backslash\{i\}$, define $t_{-i}=\left(t_{j}\right)_{j \in N \backslash\{i\}}$.

Lemma 10 Assume the conditions of Theorem 1 and suppose $b_{i}$ is defined. If $\nexists x_{-i} \in \operatorname{Int}\left(X_{-i}\right)$ such that $\left(b_{i}, x_{-i}\right) \in C^{1} \cap C^{2}$, then one of the following two cases holds.

(1) $t_{-i}$ is defined and $\left(b_{i}, t_{-i}\right) \in C^{1} \cap C^{2}$.

(2) $t_{-i}$ is not defined and $\nexists x_{-i} \in X_{-i}:\left(b_{i}, x_{-i}\right) \in C^{1} \cap C^{2}$.

Proof. We first prove that both cases are possible. Case 1: let $X=[0,1]^{3}$, $x \in C^{2}$ iff $x_{1}+x_{2}+x_{3} \geq 2$ and $x \in C^{1}$ iff $x_{1}+x_{2}+x_{3} \leq 2 ; b_{i}=0$ and $t_{i}=1$ on all dimensions; besides, $\left(b_{i}, t_{-i}\right) \in C^{1} \cap C^{2}$ for all $i$. Case 2: let $X=[0,1] \times] 0,1\left[^{2}, x \in C^{2}\right.$ iff $x_{1}+x_{2}+x_{3} \geq 2$ and $x \in C^{1}$ iff $x_{1}+x_{2}+x_{3} \leq 2$; $b_{1}=0$ but $t_{2}$ and $t_{3}$ are not defined; besides there is no $x_{-1}:\left(0, x_{-1}\right) \in C^{1} \cap C^{2}$.

We now prove that no other case is possible. We must consider three cases.

(i) $t_{-i}$ is defined and $\left(b_{i}, t_{-i}\right) \in I^{1}$. Consider any $x$ in $C^{1} \cap C^{2}$. By strict monotonicity (Lemma 4$),\left(x_{i}, t_{-i}\right) \in I^{2}$. By Restricted Solvability, there is 
$y_{i}:\left(y_{i}, t_{-i}\right) \in C^{1} \cap C^{2}$. By strict monotonicity, $b_{i} \prec_{i}^{R} y_{i}$. Clearly, there is no $z_{i}: b_{i} \prec_{i}^{R} z_{i} \prec_{i}^{R} y_{i}$. So, $X_{i}$ is not connected. A contradiction.

(ii) $t_{-i}$ is defined and $\left(b_{i}, t_{-i}\right) \in I^{2}$. Because the covering is not degenerate, there is $x_{-i}$ such that $\left(b_{i}, x_{-i}\right) \in I^{1}$. Otherwise, by Monotonicity, we would have $y \in C^{2}$ for all $y \in X$. Note that $x_{-i} \neq t_{-i}$ and $x_{-i} \notin \operatorname{Int}\left(X_{-i}\right)$. Since $X_{j}$ is connected, for each $j \neq i$, we can find $z_{j} \prec_{j}^{R} t_{j}$ and very 'close' to $t_{j}$ such that $\left(b_{i}, z_{-i}\right) \in I^{2}$. By Lemma $1,\left(b_{i}, x_{-i}\right) \in I^{1}$ and $\left(b_{i}, z_{-i}\right) \in I^{2}$ imply there is $w_{-i}$ such that $\left(b_{i}, w_{-i}\right) \in C^{1} \cap C^{2}$. By monotonicity, $w_{j} \precsim_{j}^{R} z_{j} \forall j \neq i$ and, hence, $w_{-i} \in \operatorname{Int}\left(X_{-i}\right)$. A contradiction.

(iii) $t_{-i}$ is not defined and $\exists x_{-i} \in X_{-i}:\left(b_{i}, x_{-i}\right) \in C^{1} \cap C^{2}$. Note that, for some (but not all) $j \neq i, x_{j}=t_{j}$. Let $I=\left\{j: x_{j}=t_{j}\right\}$. So, we can write $\left(b_{i}, x_{-i}\right)$ as $\left(b_{i}, t_{I}, x_{-i I}\right)$. Choose any $j \in N \backslash(I \cup\{i\})$. Since $x_{j} \neq t_{j}$, there is $y_{j} \succ_{j}^{R} x_{j}$. By strict monotonicity, $\left(b_{i}, t_{I}, x_{-i j I}, y_{j}\right) \in I^{2}$. Choose any $l^{(1)} \in I$. By connectedness, there is $y_{l^{(1)}} \prec_{l^{(1)}}^{R} t_{l^{(1)}}$ and such that $\left(b_{i}, t_{I-l^{(1)}}, y_{l^{(1)}}, x_{-i j I}, y_{j}\right) \in I^{2}$. By strict monotonicity, $\left(b_{i}, t_{I-l^{(1)}}, y_{l^{(1)}}, x_{-i I}\right) \in$ $I^{1}$. By Restricted Solvability, there is $w_{j}^{(1)}:\left(b_{i}, t_{I-l^{(1)}}, y_{l^{(1)}}, x_{-i j I}, w_{j}^{(1)}\right) \in$ $C^{1} \cap C^{2}$. Compared to the object $\left(b_{i}, t_{I}, x_{-i I}\right)$ that we started with, this one contains one less 'top' coordinate $\left(t_{l^{(1)}}\right)$ and one more interior coordinate $\left(y_{l^{(1)}}\right)$. Let us repeat this process with coordinates $j$ (the same as before) and $l^{(2)} \in I \backslash\{l\}$. We obtain $\left(b_{i}, t_{I-l^{(1)} l^{(2)}}, y_{l^{(1)} l^{(2)}}, x_{-i j I}, w_{j}^{(2)}\right) \in C^{1} \cap C^{2}$

If we repeat this process \#I times, we will finally obtain an object $\left(b_{i}, y_{I}, x_{-i j I}, w_{j}^{(\# I)}\right)$ containing no top coordinate but contained in $C^{1} \cap C^{2}$. A contradiction.

Proof of Theorem 1. If $X_{i}$ is unbounded for all $i$ in $N$, then we can directly apply Proposition 1 and we are done. We now look at the case where at least one dimension is not unbounded. Let us first restrict our attention to $\operatorname{Int}(X)$, in the product topology induced by the weak orders $\succsim_{i}^{R}$. The set $\operatorname{Int}(X)$ is the product $\prod_{i \in N} \operatorname{Int}\left(X_{i}\right)$ and $\operatorname{Int}\left(X_{i}\right)$ is unbounded for all $i$. Hence, applying Proposition 1 , we can find an additive representation $\left(u_{i}\right)_{i \in N}$ on $\operatorname{Int}(X)$. We will now extend it to $X$, i.e., we will fix, for each dimension $i$, $u_{i}\left(b_{i}\right)$ and $u_{i}\left(t_{i}\right)$, if they exist. We show how to do this only for for $b_{i}$.

If there is $x_{-i} \in \operatorname{Int}\left(X_{-i}\right):\left(b_{i}, x_{-i}\right) \in C^{1} \cap C^{2}$, then fix

$$
u_{i}\left(b_{i}\right)=s^{2}-\sum_{j \in N \backslash\{i\}} u_{j}\left(x_{j}\right) .
$$

Otherwise, by Lemma 10, one of the following two cases can arise.

(1) $t_{-i}$ is not defined and $\nexists x_{-i} \in X_{-i}:\left(b_{i}, x_{-i}\right) \in C^{1} \cap C^{2}$. We must choose $u_{i}\left(b_{i}\right)$ smaller than $u_{i}\left(x_{i}\right)$ for any $x_{i} \succ_{i}^{R} b_{i}$. We then choose $u_{i}\left(b_{i}\right)=$ $\inf \left\{u_{i}\left(x_{i}\right): x_{i} \in \operatorname{Int}\left(X_{i}\right)\right\}$ so that $u_{i}\left(X_{i}\right)$ is connected and $u_{i}$ is continuous.

(2) $t_{-i}$ is defined and $\left(b_{i}, t_{-i}\right) \in C^{1} \cap C^{2}$. Choose any $l^{(1)} \neq i$. By connect- 
edness, there is $y_{l^{(1)}} \prec_{l^{(1)}}^{R} t_{l^{(1)}}$. By strict monotonicity, $\left(b_{i}, t_{-i l^{(1)}}, y_{l^{(1)}}\right) \in$ $I^{1}$. By connectedness, there is $w_{i}^{(1)}:\left(w_{i}^{(1)}, t_{-i l^{(1)}}, y_{l^{(1)}}\right) \in I^{1}$. By strict monotonicity, $\left(w_{i}^{(1)}, t_{-i}\right) \in I^{2}$. By Restricted Solvability, there is $w_{l^{(1)}}$ : $\left(w_{i}^{(1)}, t_{-i l^{(1)}}, w_{l^{(1)}}\right) \in C^{1} \cap C^{2}$. We follow the same reasoning for dimension $l^{(2)} \neq i, l^{(1)}$ and we obtain $\left(w_{i}^{(2)}, t_{-i l^{(1)} l^{(2)}}, w_{l^{(1)} l^{(2)}}\right) \in C^{1} \cap C^{2}$. We follow the same reasoning for all coordinates but $i$ and $l$ and we finally obtain an object $\left(w_{i}^{(n-2)}, t_{l}, w_{-i l}\right) \in C^{1} \cap C^{2}$. We then fix

$$
u_{l}\left(t_{l}\right)=s^{2}-u_{i}\left(w_{i}^{(n-2)}\right)-\sum_{j \in N \backslash\{i, l\}} u_{j}\left(w_{j}\right) .
$$

We do this for all $l \neq i$. We finally take $u_{i}\left(b_{i}\right)=s^{2}-\sum_{j \in N \backslash\{i\}} u_{j}\left(t_{j}\right)$.

\section{A.4 Proof of Corollary 1}

Just as we did with Theorem 1, we first prove a result where influence of each attribute is replaced by unboundedness.

Proposition 2 Consider a structure $\left\langle X,\left(C^{1}, C^{2}\right)\right\rangle$ with $n \geq 3$ and $\left(C^{1}, C^{2}\right)$ a partition of $X$ such that $X_{i}$ is unbounded and connected in the interval topology induced by $\succsim_{i}^{R}$, for every $i \in N$. Then it has a continuous representation in the Additive Model Without Frontier iff it satisfies 1-Linearity-I, 2-Linearity-I (when $n>3$ ), Double Cancellation (when $n=3$ ) and $C^{2}$ is open in the product topology generated by the interval topology on each $X_{i}$. The mappings $u_{i}$ are unique up to $n$ positive affine transformations with the same multiplicative constant.

Proof. Consider the structure $\left\langle X,\left(C^{1}, \mathrm{Cl}\left(C^{2}\right)\right)\right\rangle$, where $\mathrm{Cl}\left(C^{2}\right)$ denotes the topological closure of $C^{2}$ in $X$. This structure is an ordered covering and it is non-degenerate because, $\left(C^{1}, C^{2}\right)$ being a partition, $C^{1} \neq \emptyset \neq C^{2}$. It is easy to check that this new structure satisfies 1-Linearity-I and 2-Linearity-I. We now prove that it also satisfies Restricted Solvability, 1-Thinness, 1-Linearity-F and Double Cancellation.

$\left\langle X,\left(C^{1}, \mathrm{Cl}\left(C^{2}\right)\right)\right\rangle$ satisfies Restricted Solvability.

Suppose $\left(x_{i}, a_{-i}\right) \in C^{1}$ and $\left(y_{i}, a_{-i}\right) \in \operatorname{Cl}\left(C^{2}\right)$. Let $V\left(a_{-i}\right)=\left\{w_{i}:\left(w_{i}, a_{-i}\right) \in\right.$ $\left.C^{1}\right\}$ and $W\left(a_{-i}\right)=\left\{w_{i}:\left(w_{i}, a_{-i}\right) \in C^{2}\right\}$. Note that $V\left(a_{-i}\right) \neq \emptyset \neq W\left(a_{-i}\right)$. By Lemma 3, $\left(w_{i}, a_{-i}\right) \succsim_{i}^{R}\left(w_{i}^{\prime}, a_{-i}\right)$ for all $w_{i} \in W\left(a_{-i}\right), w_{i}^{\prime} \in V\left(a_{-i}\right)$. Because $\left(C^{1}, C^{2}\right)$ is a partition of $X, V\left(a_{-i}\right)$ and $W\left(a_{-i}\right)$ form a partition of $X_{i}$. Since $X_{i}$ is connected, one of $V\left(a_{-i}\right), W\left(a_{-i}\right)$ must be open and the other one closed. Because $C^{2}$ is open, it is clear that $W\left(a_{-i}\right)$ is open, and, hence, $V\left(a_{-i}\right)$ is closed. Let $W^{\prime}\left(a_{-i}\right)=\left\{w_{i}:\left(w_{i}, a_{-i}\right) \in \mathrm{Cl}\left(C^{2}\right)\right\}$. We have $W\left(a_{-i}\right) \subseteq W^{\prime}\left(a_{-i}\right)$. 
If $W\left(a_{-i}\right) \subset W^{\prime}\left(a_{-i}\right)$, then $V\left(a_{-i}\right) \cap W^{\prime}\left(a_{-i}\right) \neq \emptyset$ and Restricted Solvability holds. If $W\left(a_{-i}\right)=W^{\prime}\left(a_{-i}\right)$, then $W\left(a_{-i}\right)$ is closed but we have shown that this is not possible.

$\left\langle X,\left(C^{1}, \mathrm{Cl}\left(C^{2}\right)\right)\right\rangle$ satisfies 1-Thinness.

Suppose $\left(x_{i}, a_{-i}\right) \in C^{1} \cap \mathrm{Cl}\left(C^{2}\right)$. As shown above, $X_{i}$ can be written as $V\left(a_{-i}\right) \cup W\left(a_{-i}\right)$. Since $V\left(a_{-i}\right)$ is closed and $W\left(a_{-i}\right) \neq \emptyset, \exists z_{i}: z_{i} \succsim_{i}^{R} w_{i} \forall w_{i} \in$ $V\left(a_{-i}\right)$. So, $V\left(a_{-i}\right)=\left\{w_{i}: w_{i} \precsim_{i}^{R} z_{i}\right\}$ and $W\left(a_{-i}\right)=\left\{w_{i}: w_{i} \succ_{i}^{R} z_{i}\right\}$. It is easily seen that $W^{\prime}\left(a_{-i}\right)=\operatorname{Cl}\left(W\left(a_{-i}\right)\right)=\left\{w_{i}: w_{i} \succsim_{i}^{R} z_{i}\right\}$. So, $x_{i} \sim_{i}^{R} z_{i}$. Similarly, $\left(y_{i}, a_{-i}\right) \in C^{1} \cap \operatorname{Cl}\left(C^{2}\right)$ implies $x_{i} \sim_{i}^{R} z_{i} \sim_{i}^{R} y_{i}$ and, by definition of $\succsim_{i}^{R},\left(x_{i}, b_{-i}\right) \in C^{1} \cap \mathrm{Cl}\left(C^{2}\right)$ and $\left(y_{i}, b_{-i}\right) \in C^{1} \cap \mathrm{Cl}\left(C^{2}\right)$.

$\left\langle X,\left(C^{1}, \mathrm{Cl}\left(C^{2}\right)\right)\right\rangle$ satisfies 1-Linearity-F.

Suppose

$$
\begin{aligned}
\left(x_{i}, a_{-i}\right) & \in C^{1} \cap \mathrm{Cl}\left(C^{2}\right),\left(y_{i}, a_{-i}\right) \notin \mathrm{Cl}\left(C^{2}\right), \\
\left(y_{i}, b_{-i}\right) & \in C^{1} \cap \mathrm{Cl}\left(C^{2}\right),\left(x_{i}, b_{-i}\right) \notin \mathrm{Cl}\left(C^{2}\right) .
\end{aligned}
$$

From (A.10) and the discussion about Restricted Solvability (two paragraphs earlier), we find $x_{i} \succ_{i} y_{i}$. From (A.11), we find $y_{i} \succ_{i} x_{i}$. A contradiction. $\diamond$

\section{$\left\langle X,\left(C^{1}, \mathrm{Cl}\left(C^{2}\right)\right)\right\rangle$ satisfies Double Cancellation.}

We need to check this only for $n=3$. If $x \in \mathrm{Cl}\left(C^{2}\right) \backslash C^{1}$, then $x \in C^{2}$. So, $\left\langle X,\left(C^{1}, \mathrm{Cl}\left(C^{2}\right)\right)\right\rangle$ violates Double Cancellation if there are $x_{i}, y_{j}, x_{h}, y_{i}, z_{j}, y_{h}, z_{i}, x_{j}, z_{h}$ such that

$$
\begin{array}{ll}
\left(x_{i}, y_{j}, x_{h}\right) \in C^{2}, & \left(y_{i}, x_{j}, x_{h}\right) \notin \mathrm{Cl}\left(C^{2}\right), \\
\left(y_{i}, z_{j}, y_{h}\right) \in C^{2}, & \left(z_{i}, y_{j}, y_{h}\right) \notin \mathrm{Cl}\left(C^{2}\right), \\
\left(z_{i}, x_{j}, z_{h}\right) \in C^{2}, & \left(x_{i}, z_{j}, z_{h}\right) \notin \mathrm{Cl}\left(C^{2}\right) .
\end{array}
$$

The set $X_{h}$ being unbounded and connected, there are $x_{h}^{\prime} \succ_{h}^{R} x_{h}, y_{h}^{\prime} \succ_{h}^{R} y_{h}$ and $z_{h}^{\prime} \succ_{h}^{R} z_{h}$ such that $\left(y_{i}, x_{j}, x_{h}^{\prime}\right) \in C^{1},\left(z_{i}, y_{j}, y_{h}^{\prime}\right) \in C^{1}$ and $\left(x_{i}, z_{j}, z_{h}\right) \in C^{1}$. By strict monotonicity, $\left(x_{i}, y_{j}, x_{h}^{\prime}\right) \in C^{2},\left(y_{i}, z_{j}, y_{h}^{\prime}\right) \in C^{2}$ and $\left(z_{i}, x_{j}, z_{h}^{\prime}\right) \in C^{2}$. Hence, $\left\langle X,\left(C^{1}, C^{2}\right)\right\rangle$ violates Double Cancellation. A contradiction.

So, by Theorem 1 , there is a representation $\left\langle\left(u_{i}\right)_{i \in N},\left(s^{k}\right)_{k=1, \ldots, 3}\right\rangle$ of $\left\langle X,\left(C^{1}, \mathrm{Cl}\left(C^{2}\right)\right)\right\rangle$ in the Additive Model With Frontier. It is then clear that $\left\langle\left(u_{i}\right)_{i \in N},\left(s^{k}\right)_{k=1, \ldots, 3}\right\rangle$ is also a representation of $\left\langle X,\left(C^{1}, C^{2}\right)\right\rangle$ in the Additive Model Without Frontier. 
Proof of Corollary 1. We can now replicate the reasoning that we used in Section A.3 (proof of Lemma 10 and Theorem 1). The only difference is that we everywhere replace $C^{2}$ by $\mathrm{Cl}\left(C^{2}\right)$.

\section{A.5 Proof of Theorem 2}

Our strategy for proving Theorem 2 will be to first prove the result when $X_{i}$ is unbounded for all $i \in N$. We will then handle the general case. But, first, some notation and two lemmas.

For any $k \in R^{+}$and $i \in N$, define the relation $\succsim_{i}^{k}$ on $X_{i}^{k}$ by $x_{i} \succsim_{i}^{k} y_{i}$ iff, $\forall a_{-i} \in X_{-i}$

- $\left(y_{i}, a_{-i}\right) \in C^{k} \cap C^{k-1}$ implies $\left(x_{i}, a_{-i}\right) \in C_{\geq}^{k}$ and

- $\left(y_{i}, a_{-i}\right) \in I^{k}$ implies $\left(x_{i}, a_{-i}\right) \in I_{\geq}^{k}$.

Lemma 11 Assume all conditions of Theorem 2. Then, for any $k \in R^{+}$, the restriction of $\succsim_{i}^{R}$ to $X_{i}^{k}$ is identical to $\succsim_{i}^{k}$ for all $i \in N$. In particular, $\succsim_{i}^{R}=\succsim_{i}^{l}$.

Proof. Choose $x_{i}$ and $y_{i}$ in $X_{i}^{k}$. Suppose $x_{i} \succsim_{i}^{R} y_{i}$. Then, it follows easily from the definition of $\succsim_{i}^{R}$ and $\succsim_{i}^{k}$ that $x_{i} \succsim_{i}^{k} y_{i}$. Suppose now that $x_{i} \succsim_{i}^{k} y_{i}$ and, contrary to what we must prove, $y_{i} \succ_{i}^{R} x_{i}$. Then we must have $x_{i} \sim_{i}^{k} y_{i}$ and $y_{i} \succ_{i}^{k^{\prime}} x_{i}$ for some $k^{\prime} \neq k$. The indifference $x_{i} \sim_{i}^{k} y_{i}$ implies the existence of $a_{-i}$ such that $\left(x_{i}, a_{-i}\right) \in C^{k-1} \cap C^{k}$ and $\left(y_{i}, a_{-i}\right) \in C^{k-1} \cap C^{k}$. Then, by 1Thinness, $\left(x_{i}, b_{-i}\right) \in C_{\geq}^{k^{\prime}}$ iff $\left(y_{i}, b_{-i}\right) \in C_{\geq}^{k^{\prime}}$ and $\left(x_{i}, b_{-i}\right) \notin I_{\geq}^{k^{\prime}}$ iff $\left(y_{i}, b_{-i}\right) \notin I_{\geq}^{k^{\prime}}$. This contradicts the fact that $y_{i} \succ_{i}^{k^{\prime}} x_{i}$ and concludes the proof.

Lemma 12 Assume all conditions of Theorem 2 and that $X_{i}$ is unbounded for all $i \in N$. Then $\left(x_{i j}, a_{-i j}\right) \in C^{k-1} \cap C^{k},\left(y_{i j}, b_{-i j}\right) \in C^{l-1} \cap C^{l}$ and $\left(y_{i j}, a_{-i j}\right) \in C^{k-1} \cap C^{k} i m p l y\left(x_{i j}, b_{-i j}\right) \in C^{l-1} \cap C^{l}$.

Proof. Suppose $\left(x_{i j}, a_{-i j}\right) \in C^{k-1} \cap C^{k},\left(y_{i j}, b_{-i j}\right) \in C^{l-1} \cap C^{l}$ and $\left(y_{i j}, a_{-i j}\right) \in$ $C^{k-1} \cap C^{k}$ but $\left(x_{i j}, b_{-i j}\right) \notin C^{l-1} \cap C^{l}$. Two cases can arise: $\left(x_{i j}, b_{-i j}\right) \notin C_{\geq}^{l}$ or $\left(x_{i j}, b_{-i j}\right) \in I_{\geq}^{l}$. Suppose first $\left(x_{i j}, b_{-i j}\right) \notin C_{\geq}^{l}$. By Connectedness, there is $x_{i}^{\prime} \succ_{i}^{R} x_{i}$ such that $\left(x_{i}^{\prime}, x_{j}, b_{-i j}\right) \notin C_{\geq}^{l}$. By strict monotonicity (Lemma 4), $\left(x_{i}^{\prime}, x_{j}, a_{-i j}\right) \in I_{\geq}^{k}$. By connectedness and unboundedness, there is $b_{h}^{\prime} \succ_{h}^{R} b_{h}$ $(i \neq h \neq j)$ such that $\left(x_{i}^{\prime}, x_{j}, b_{h}^{\prime}, b_{-i j h}\right) \notin C_{\geq}^{l}$. By Lemma $4,\left(y_{i j}, b_{h}^{\prime}, b_{-i j h}\right) \in I_{\geq}^{l}$. This contradicts 2-Linearity-I.

Suppose now $\left(x_{i j}, b_{-i j}\right) \in I_{\geq}^{l}$. This case is handled like the previous one. So, $\left(x_{i j}, b_{-i j}\right) \in C^{l-1} \cap C^{l}$. 
Proof of Theorem 2. We first handle the unbounded case. By definition, $X^{k}$ is a Cartesian product. The sets $C^{k, 2}$ and $C^{k, 1}$ form a covering of $X^{k}$ and clearly satisfy all conditions of Proposition 1 . Hence, for $k \in R^{+}$, there is an additive representation $\left\langle\left(v_{i}^{k}\right)_{i \in N}\right\rangle$ of $\left\langle X^{k},\left(C^{k, 2}, C^{k, 1}\right)\right\rangle$ such that, for every $k \in R^{+}$, we have

$$
\begin{aligned}
& \sum_{i=1}^{n} v_{i}^{k}\left(x_{i}\right) \geq s^{k} \Leftrightarrow x \in C^{k, 2}, \\
& \sum_{i=1}^{n} v_{i}^{k}\left(x_{i}\right) \leq s^{k} \Leftrightarrow x \in C^{k, 1} .
\end{aligned}
$$

By construction, in each representation $\left\langle\left(v_{i}^{k}\right)_{i \in N}\right\rangle$, the frontier between $C^{k, 2}$ and $C^{k, 1}$ is a (hyper)plane with slope -1 in all directions.

Thanks to Overlap, we know that $X^{k} \subseteq X^{l}=X$. For the sake of definiteness, suppose $k>l$ (if $k<l$, a symmetric reasoning can be used). We have seen in Lemma 11 that $v_{i}^{l}$ and $v_{i}^{k}$ are two continuous representations of the same relation $\succsim_{i}^{R}$ (restricted to $X_{i}^{k}$ ), for all $i \in N$. Hence, they are identical up to a continuous strictly increasing transformation. So, because the image of $C^{k-1} \cap C^{k}$ through $\left\langle\left(v_{i}^{k}\right)_{i \in N}\right\rangle$ is a hyperplane, its image through $\left\langle\left(v_{i}^{l}\right)_{i \in N}\right\rangle$ is a connected strictly decreasing hypersurface lying above the image of $C^{l-1} \cap C^{l}$. Actually, we will show that the image of $C^{k-1} \cap C^{k}$ through the representation $\left\langle\left(v_{i}^{l}\right)_{i \in N}\right\rangle$ is also a (hyper)plane, parallel to the one representing the frontier between $C^{l}$ and $C^{l-1}$.

Consider the set $E_{i}^{k}=\left\{x_{i} \in X_{i}:\left(y_{-i}, x_{i}\right) \in C^{k-1} \cap C^{k}\right.$ for some $y_{-i} \in X_{-i}$ and $\left(y_{-i}, y_{i}^{\prime}\right) \in C^{l-1} \cap C^{l}$ for some $y_{i}^{\prime} \in X_{i}$. This set has no minimal element, because, all $X_{j}$ being unbounded, there is no minimal $y_{i}^{\prime}:\left(y_{-i}, y_{i}^{\prime}\right) \in C^{l-1} \cap C^{l}$. It is not empty because, by Overlap, it contains $x_{i}^{(i)}$. Notice that $x_{i} \in E_{i}^{k}$ and $z_{i} \succ_{i}^{R} x_{i}$ imply $z_{i} \in E_{i}^{k}$ (because the image of $C^{k-1} \cap C^{k}$ lies above that of $\left.C^{l-1} \cap C^{l}\right)$.

Choose some $z_{i} \in E_{i}^{k}$. There is $z_{-i} \in X_{-i}: z \in C^{k-1} \cap C^{k}$ and there is $z_{i}^{\prime} \in$ $X_{i}:\left(z_{i}^{\prime}, z_{-i}\right) \in C^{l-1} \cap C^{l}$. Choose now any $j, h \neq i$. For all $w_{j h}:\left(w_{j h}, z_{-j h}\right) \in$ $C^{k-1} \cap C^{k}$, Lemma 12 implies $\left(w_{j h}, z_{-i j h}, z_{i}^{\prime}\right) \in C^{l-1} \cap C^{l}$. So, for all $w_{j h}$ : $\left(w_{j h}, z_{-j h}\right) \in C^{k-1} \cap C^{k}$,

$$
\begin{gathered}
v_{j}^{l}\left(w_{j}\right)+v_{h}^{l}\left(w_{h}\right)+\sum_{g \in N \backslash\{j, h\}} v_{g}^{l}\left(z_{g}\right) \\
=v_{j}^{l}\left(w_{j}\right)+v_{h}^{l}\left(w_{h}\right)+\sum_{g \in N \backslash\{i, j, h\}} v_{g}^{l}\left(z_{g}\right)+v_{i}^{l}\left(z_{i}^{\prime}\right)+\kappa=\sigma^{l}+\kappa,
\end{gathered}
$$

where $\kappa=v_{i}^{l}\left(z_{i}\right)-v_{i}^{l}\left(z_{i}^{\prime}\right)$. So, for all $w_{j h}:\left(w_{j h}, z_{-j h}\right) \in C^{k-1} \cap C^{k}$, the $\operatorname{sum} v_{j}^{l}\left(w_{j}\right)+v_{h}^{l}\left(w_{h}\right)+\sum_{g \in N \backslash\{j, h\}} v_{g}^{l}\left(z_{g}\right)$ is constant. This implies that the image of the set $d_{j h}(z)=\left\{\left(w_{j h}, z_{-j h}\right) \in C^{k-1} \cap C^{k}\right\}$ is a straight line in the representation $\left\langle\left(v_{i}^{l}\right)_{i \in N}\right\rangle$, for any $z \in C^{k-1} \cap C^{k}$ with $z_{i} \in E_{i}^{k}$ and $j, h \neq i$. Let $e_{i}(z)$ be defined as $\bigcup_{j, h \neq i} d_{j h}(z)$. Since all sets in $e_{i}(z)$ have the point $z$ in 
common, the image of the set $e_{i}(z)$ lies in a hyperplane with $n-1$ dimensions in the representation $\left\langle\left(v_{i}^{l}\right)_{i \in N}\right\rangle$.

Remark that, for any $w \in e_{i}(z), w$ differs from $z$ on exactly two dimensions (or zero if $w=z$ ). Remark also that, for any $w \in e_{i}(z)$, we can define $e_{i}(w)$ and show that the image of the set $e_{i}(w)$ through the representation $\left\langle\left(v_{i}^{l}\right)_{i \in N}\right\rangle$ lies in a hyperplane: the same one as $e_{i}(z)$ because $w \in e_{i}(z) \cap e_{i}(w)$. For any $w^{\prime} \in e_{i}(w), w^{\prime}$ differs from $z$ on two, three or four dimensions (or zero if $\left.w^{\prime}=z\right)$ and we can define $e_{i}\left(w^{\prime}\right)$ and show that the image of the set $e_{i}\left(w^{\prime}\right)$ through the representation $\left\langle\left(v_{i}^{l}\right)_{i \in N}\right\rangle$ lies in the same hyperplane as $e_{i}(z)$. For any $w^{\prime \prime} \in e_{i}\left(w^{\prime}\right), w^{\prime \prime}$ differs from $z$ on two, three, four, five or six dimensions (or zero if $w^{\prime \prime}=z$ ). Going on like this, we see that, starting from $z$, we can reach points differing from $z$ on any number of dimensions between 2 and $n$. Now, is there $w_{-i}^{*} \in X_{-i}$ such that $\left(z_{i}, w_{-i}^{*}\right) \in C^{k-1} \cap C^{k}$ and $\left(z_{i}, w_{-i}^{*}\right)$ cannot be reached from $z$ in the same way as above? The answer is 'no'. Indeed, remember that $C^{k-1} \cap C^{k}$ has an additive representation, so that the image of $C^{k-1} \cap C^{k}$ through the representation $\left\langle\left(v_{i}^{k}\right)_{i \in N}\right\rangle$ is a hyperplane in the image of $X^{k}$. The image of the set of all points $\left(z_{i}, w_{-i}^{*}\right) \in C^{k-1} \cap C^{k}$ (with $z_{i}$ fixed) through the representation $\left\langle\left(v_{i}^{k}\right)_{i \in N}\right\rangle$ is therefore a hyperplane in the image of $z_{i} \times X_{-i}^{k}$. So, starting from $z$ in this hyperplane, we can reach any other point of this hyperplane in a finite number of moves as described above (the image of each move being a line segment). We therefore conclude that the image of the set $g_{i}\left(z_{i}\right)=\bigcup_{z_{-i}: z \in C^{k-1} \cap C^{k}} e_{i}(z)$ through the representation $\left\langle\left(v_{i}^{l}\right)_{i \in N}\right\rangle$ lies in a hyperplane with $n-1$ dimensions, for any $z_{i} \in E_{i}^{k}$ and any $i \in N$.

Define finally $D_{i}=\bigcup_{z_{i} \in E_{i}^{k}} g_{i}\left(z_{i}\right)$. Since two sets $g_{i}\left(z_{i}\right)$ and $g_{i}\left(z_{i}^{\prime}\right)$ have no points in common, we do not know yet whether $D_{i}$ lies in a hyperplane. We have to prove this. We do it by showing that, for any $z_{i}, z_{i}^{\prime} \in E_{i}^{k}$ and $j \in N$, there are two sequences $w_{j}^{(1)}, w_{j}^{(2)}, w_{j}^{(3)}, \ldots, w_{j}^{(q)}$ and $z_{i}^{(2)}, z_{i}^{(3)}, \ldots, z_{i}^{(q)}$ with $q \geq 1^{5}$ such that $g_{i}\left(z_{i}\right) \cap g_{j}\left(w_{j}^{(1)}\right) \neq \emptyset, g_{i}\left(z_{i}^{\prime}\right) \cap g_{j}\left(w_{j}^{(q)}\right) \neq \emptyset, g_{j}\left(w_{j}^{(h)}\right) \cap g_{i}\left(z_{i}^{(h+1)}\right) \neq \emptyset \neq$ $g_{j}\left(w_{j}^{(h+1)}\right) \cap g_{i}\left(z_{i}^{(h+1)}\right)$, for all $h: 1 \leq h<q-1$. We consider two cases (see fig. A.1).

(a) For some $h \neq i$, there is $y_{h}$ such that $\left(y_{h}, y_{-h}\right) \notin C^{k-1} \cap C^{k}$ for all $y_{-h} \in X_{-h}$. Then, clearly, for any $z_{i}, z_{i}^{\prime} \in E_{i}^{k}$ and $j \in N$, there is $w_{j}^{(1)} \in E_{j}^{k}$ such that $g_{j}\left(w_{j}^{(1)}\right)$ intersects $g_{i}\left(z_{i}\right)$ and $g_{i}\left(z_{i}^{\prime}\right)$. So, in this case, $q=1$.

(b) For some $h \neq i$, for some $y_{h}$ and for all $z_{h}: z_{h} \prec_{h}^{R} y_{h}$, there is $w_{-h}$ such that $\left(z_{h}, w_{-h}\right) \in C^{k-1} \cap C^{k}$. It can then happen (as in fig. A.1) that, for some $j \in N$, there is no $w_{j}^{(1)} \in E_{j}^{k}$ such that $g_{j}\left(w_{j}^{(1)}\right)$ intersects $g_{i}\left(z_{i}\right)$ and $g_{i}\left(z_{i}^{\prime}\right)$. We then need to use a sequence with $q>1$. Notice first that, for any $x_{i} \in E_{i}^{k}$, there is $w_{j} \in E_{j}^{k}$ such that $g_{j}\left(w_{j}\right)$ intersects $g_{i}\left(x_{i}\right)$. Similarly, for any $x_{j} \in E_{j}^{k}$, there is $w_{i} \in E_{i}^{k}$ such that $g_{i}\left(w_{i}\right)$ intersects $g_{j}\left(x_{j}\right)$. So,

$\overline{5 \text { If } q=1}$, the second sequence is not defined 
starting from $z_{i}$, we find $w_{j}^{(1)}$ such that $g_{j}\left(w_{j}^{(1)}\right)$ intersects $g_{i}\left(z_{i}\right)$. If $g_{j}\left(w_{j}^{(1)}\right)$ does not intersect $g_{i}\left(z_{i}^{\prime}\right)$, we find $z_{i}^{(2)}$ such that $g_{j}\left(w_{j}^{(1)}\right)$ intersects $g_{i}\left(z_{i}^{(2)}\right)$. We then find $w_{j}^{(2)}$ such that $g_{j}\left(w_{j}^{(2)}\right)$ intersects $g_{i}\left(z_{i}^{(2)}\right)$. If $g_{j}\left(w_{j}^{(2)}\right)$ does not intersect $g_{i}\left(z_{i}^{\prime}\right)$, we find $z_{i}^{(3)}$ such that $g_{j}\left(w_{j}^{(2)}\right)$ intersects $g_{i}\left(z_{i}^{(3)}\right)$. And so on. At each iteration of this process, $z_{i}^{(h)}$ comes closer and closer to $z_{i}^{\prime}$. In the representation $\left\langle\left(v_{i}^{k}\right)_{i \in N}\right\rangle$, the distance between $z_{i}^{(h)}$ and $z_{i}^{\prime}$ decreases by a finite quantity at each step and we can manage to hold this quantity constant. So, after some iterations, $z_{i}^{(h)}$ is so close to $z_{i}^{\prime}$ that we are sure we can find $w_{j}^{(h)}$ intersecting $g_{i}\left(z_{i}^{\prime}\right)$.

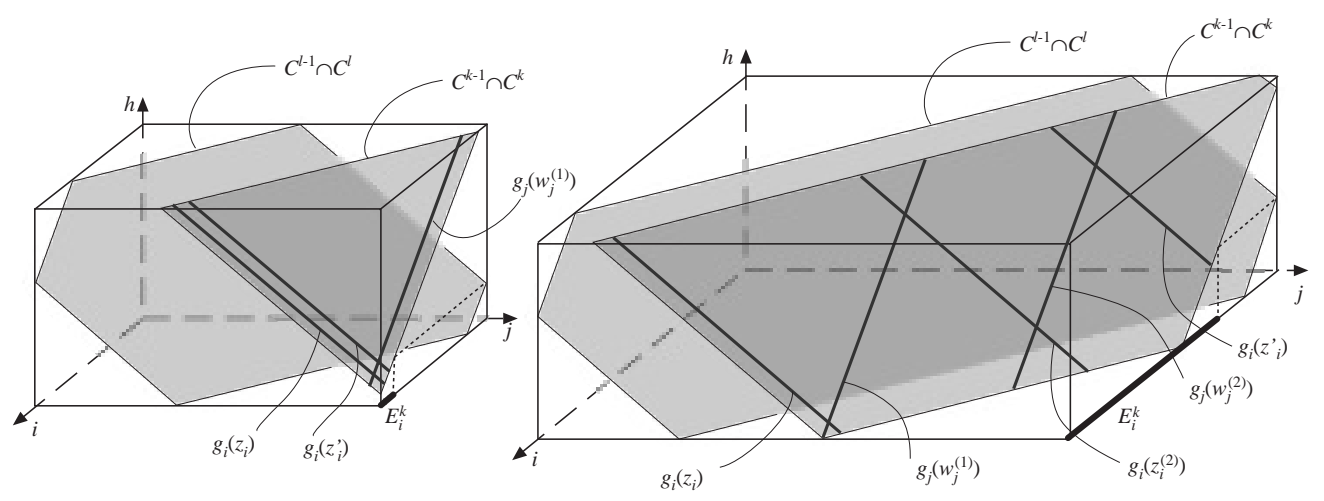

Fig. A.1. Images of $C^{k-1} \cap C^{k}$ and $C^{l-1} \cap C^{l}$ through $\left\langle\left(v_{i}^{l}\right)_{i \in N}\right\rangle$. Left: case (a). Right: case (b).

Consider now two sets $g_{i}\left(z_{i}\right)$ and $g_{i}\left(z_{i}^{\prime}\right)$ with $z_{i}, z_{i}^{\prime} \in E_{i}^{k}$. These sets have no point in common but we have just proven that they are 'linked' by the sequences $w_{j}^{(1)}, w_{j}^{(2)}, w_{j}^{(3)}, \ldots, w_{j}^{(q)}$ and $z_{i}^{(2)}, z_{i}^{(3)}, \ldots, z_{i}^{(q)}$ So, $\sum_{i \in N} v_{i}^{l}\left(x_{i}\right)$ is the same for all points $x$ in $g_{i}\left(z_{i}\right), g_{i}\left(z_{i}^{\prime}\right), g_{j}\left(w_{j}^{(h)}\right)$ and $g_{i}\left(z_{i}^{(h)}\right)$. Since this holds for all $z_{i}, z_{i}^{\prime} \in E_{i}^{k}$, the image of the set $D_{i}$ through the representation $\left\langle\left(v_{i}^{l}\right)_{i \in N}\right\rangle$ lies in a hyperplane. It clearly intersects $D_{j}$. Finally, the image of $D=\bigcup_{i \in N} D_{i}$ through the representation $\left\langle\left(v_{i}^{l}\right)_{i \in N}\right\rangle$ lies in a hyperplane (see fig. A.2).

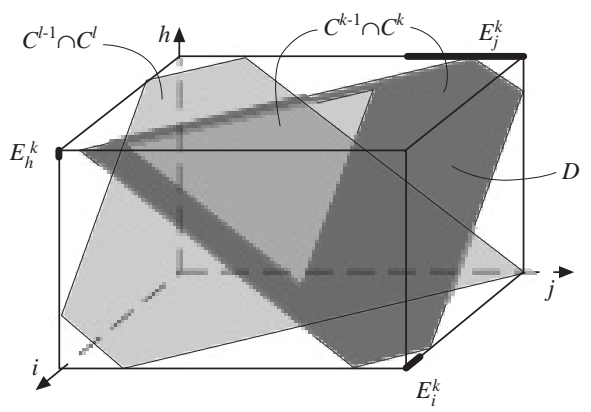

Fig. A.2. Image of the set $D$ through $\left\langle\left(v_{i}^{l}\right)_{i \in N}\right\rangle$.

Choose now one dimension, say $i^{*}$. Define $G=E_{i^{*}}^{k} \times \prod_{j \neq i^{*}} X_{j}^{k}, H^{1}=C^{k} \cap G$ and $H^{2}=C^{k+1} \cap G$. The structure $\left\langle G,\left(H^{1}, H^{2}\right)\right\rangle$ satisfies all conditions of 
Proposition 1. Hence, it has an additive representation. Actually, we have already found two such representations: one is $\left(v_{i}^{k}\right)_{i \in N}$, restricted to $G$ and the other one is $\left(v_{i}^{l}\right)_{i \in N}$, also restricted to $G$. Because of the uniqueness statement of Theorem 1, these two representations are related by positive affine transformations. So, for all $i \neq i^{*}, v_{i}^{k}$ is a positive affine transformation of $v_{i}^{l}$ (restricted to $X_{i}^{k}$ ). The mapping $v_{i^{*}}^{k}$ is also a positive affine transformation of $v_{i^{*}}^{l}$, but restricted to $E_{i}^{k}$. If we know repeat the same reasoning with dimension $j^{*}$ instead of $i^{*}$, we find that $v_{i}^{k}$ is a positive affine transformation of $v_{i}^{l}$ (restricted to $X_{i}^{k}$ ), for all $i$ in $N$. This implies that the image of $C^{k-1} \cap C^{k}$ trhough $\left(v_{i}^{l}\right)_{i \in N}$ lies in a hyperplane: the same as the one in which $D$ lies. This concludes the proof of the unbounded case.

If $X_{i}$ is not unbounded for some $i$ in $N$, then we first restrict our attention to $\operatorname{Int}(X)$, in the product topology induced by the weak orders $\succsim_{i}^{R}$. The set $\operatorname{Int}(X)$ is the product $\prod_{i \in N} \operatorname{Int}\left(X_{i}\right)$ and $\operatorname{Int}\left(X_{i}\right)$ is unbounded for all $i$. Hence, as shown above, we can find an additive representation $\left(v_{i}^{l}\right)_{i \in N}$ on $\operatorname{Int}(X)$. Because $\succsim_{i}^{R}=\succsim_{i}^{l}$, we know that $\operatorname{Int}\left(X_{i}\right)$ with respect to $\succsim_{i}^{R}$ is identical to $\operatorname{Int}\left(X_{i}\right)$ with respect to $\succsim_{i}^{l}$. So, we can extend the additive representation $\left(v_{i}^{l}\right)_{i \in N}$ on $\operatorname{Int}(X)$ to $X$ just like in Theorem 1 , using $C^{l-1} \cap C^{l}$ instead of $C^{1} \cap C^{2}$.

So far, we know that

- $x \in C^{k} \Longleftrightarrow s^{k} \leq \sum_{i \in N} v_{i}^{l}\left(x_{i}\right) \leq s^{k+1}$, for all $x \in \operatorname{Int}(X)$ and all $k \in R$;

- $x \in C_{\geq}^{l} \Longleftrightarrow s^{l} \leq \sum_{i \in N} v_{i}^{l}\left(x_{i}\right)$, for all $x \notin \operatorname{Int}(X)$;

- $x \notin I_{\geq}^{l^{-}} \Longleftrightarrow \sum_{i \in N} v_{i}^{l}\left(x_{i}\right) \leq s^{l}$, for all $x \notin \operatorname{Int}(X)$.

We still have to prove that $x \in C^{k} \Longleftrightarrow s^{k} \leq \sum_{i \in N} v_{i}^{l}\left(x_{i}\right) \leq s^{k+1}$, for all $x \notin \operatorname{Int}(X)$ and all $k \in R^{+} \backslash\{l\}$.

Suppose $x \notin \operatorname{Int}(X)$ and $x \in C^{k-1} \cap C^{k}$ with $k \neq l$. Let us show that $\sum_{i \in N} v_{i}^{l}\left(x_{i}\right)=s^{k}$. The proof will be complete if we show that $C^{k-1} \cap C^{k}$ is connected. Indeed, since the functions $v_{i}^{l}$ are continuous, the image of the connected set $C^{k-1} \cap C^{k}$ through $\sum_{i \in N} v_{i}^{l}$ is also a connected set. Hence, supposing that $\sum_{i \in N} v_{i}^{l}\left(x_{i}\right) \neq s^{k}$ would lead to a contradiction. So, let us prove that $C^{k-1} \cap C^{k}$ is connected. We have proved above (at the beginning of the proof of Proposition 1) that, when there are two categories, the set $C^{1} \cap C^{2}$ is a connected set in the unbounded case. It is easy to check that this proof also works when some $X_{i}$ is not unbounded. Hence, we can use a similar proof to show that $C^{k-1} \cap C^{k}$ is a connected set with respect to the topology on $X^{k}$ generated by the relations $\succsim_{i}^{k}$. Using Lemma 11, we know that $\succsim_{i}^{k}$ is identical to $\succsim_{i}^{l}=\succsim_{i}^{R}$ on $X_{i}^{k}$. Hence, $C^{k-1} \cap C^{k}$ is connected in the topology induced on $X$ by the relations $\succsim_{i}^{R}$.

Suppose now $x \notin \operatorname{Int}(X), \sum_{i \in N} v_{i}^{l}\left(x_{i}\right)=s^{k}$ and, contrary to what we must prove, $x \notin C^{k-1} \cap C^{k}$. 
- Suppose $x \in I_{\geq}^{k}$.

(1) Suppose $x$ has one and only one extremal coordinate $j$, that is $x_{j} \sim_{j}^{R} t_{j}$ or $x_{j} \sim_{j}^{R} b_{j}$.

- Suppose $x_{j} \sim_{j}^{R} t_{j}$. Choose any $i \neq j$. There is $y_{i} \succ_{i}^{R} x_{i}$ and $y_{j} \prec_{j}^{R} x_{j}$ such that $v_{i}^{l}\left(y_{i}\right)+v_{j}^{l}\left(y_{j}\right)+\sum_{m \in N \backslash\{i, j\}} v_{m}^{l}\left(x_{m}\right)=s^{k}$. Because $\left(y_{i}, y_{j}, x_{-i j}\right)$ has no extremal coordinate, we know that $\left(y_{i}, y_{j}, x_{-i j}\right) \in C^{k-1} \cap C^{k}$. By strict monotonicity, $\left(x_{i}, y_{j}, x_{-i j}\right) \notin C_{\geq}^{k}$. By Restricted Solvability, there is $z_{j}:\left(x_{i}, z_{j}, x_{-i j}\right) \in C^{k-1} \cap C^{k}$. But this is not possible because $v_{j}^{l}\left(z_{j}\right)+\sum_{m \in N \backslash\{j\}} v_{m}^{l}\left(x_{m}\right) \neq s^{k}$.

- Suppose $x_{j} \sim_{j}^{R} b_{j}$. Choose any $i \neq j$. There is $y_{i} \prec_{i}^{R} x_{i}$ and $y_{j} \succ_{j}^{R} x_{j}$ such that $v_{i}^{l}\left(y_{i}\right)+v_{j}^{l}\left(y_{j}\right)+\sum_{m \in N \backslash\{i, j\}} v_{m}^{l}\left(x_{m}\right)=s^{k}$. Because $\left(y_{i}, y_{j}, x_{-i j}\right)$ has no extremal coordinate, we know that $\left(y_{i}, y_{j}, x_{-i j}\right) \in C^{k-1} \cap C^{k}$. By strict monotonicity, $\left(x_{i}, y_{j}, x_{-i j}\right) \in I_{\geq}^{k}$. By Restricted Solvability, there is $z_{j}:\left(x_{i}, z_{j}, x_{-i j}\right) \in C^{k-1} \cap C^{k}$. But this is not possible because $v_{j}^{l}\left(z_{j}\right)+\sum_{m \in N \backslash\{j\}} v_{m}^{l}\left(x_{m}\right) \neq s^{k}$.

(2) Suppose $x$ has exactly two extremal coordinates $i$ and $j$. There are then four possible cases: (1) $x_{i} \sim_{i}^{R} t_{i}$ and $x_{j} \sim_{j}^{R} t_{j}$, (2) $x_{i} \sim_{i}^{R} t_{i}$ and $x_{j} \sim_{j}^{R} b_{j}$, (3) $x_{i} \sim_{i}^{R} b_{i}$ and $x_{j} \sim_{j}^{R} t_{j}$, (4) $x_{i} \sim_{i}^{R} b_{i}$ and $x_{j} \sim_{j}^{R} b_{j}$. Let us first handle case (1). Choose any $h \neq i, j$. There is $y_{h} \succ_{h}^{R} x_{h}$ and $y_{j} \prec_{j}^{R} x_{j}$ such that $v_{j}^{l}\left(y_{j}\right)+v_{h}^{l}\left(y_{h}\right)+\sum_{m \in N \backslash\{j, h\}} v_{m}^{l}\left(x_{m}\right)=s^{k}$. Because $\left(y_{j}, y_{h}, x_{-j h}\right)$ has only one extremal coordinate, we know that $\left(y_{j}, y_{h}, x_{-j h}\right) \in C^{k-1} \cap C^{k}$. By strict monotonicity, $\left(y_{j}, x_{h}, x_{-i j}\right) \notin C_{\geq}^{k}$. By Restricted Solvability, there is $z_{j}:\left(z_{j}, x_{h}, x_{-j h}\right) \in C^{k-1} \cap C^{k}$. But this is not possible because $v_{j}^{l}\left(z_{j}\right)+$ $\sum_{m \in N \backslash\{j\}} v_{m}^{l}\left(x_{m}\right) \neq s^{k}$. The three other cases lead to a contradiction in the same way.

(3) We can iterate the same reasoning for three, four, five, ... extremal coordinates.

(4) When all coordinates are extremal, three cases must be considered: they are all maximal, all minimal or some are minimal and some are maximal. The first two cases are impossible because $I^{k}$ or $I^{k-1}$ would be empty. In the third case, we choose $h$ and $j$ such that $x_{h} \sim_{h}^{R} b_{h}$ and $x_{j} \sim_{j}^{R}$ $t_{j}$. There is then $y_{h} \succ_{h}^{R} x_{h}$ and $y_{j} \prec_{j}^{R} x_{j}$ such that $v_{j}^{l}\left(y_{j}\right)+v_{h}^{l}\left(y_{h}\right)+$ $\sum_{m \in N \backslash\{j, h\}} v_{m}^{l}\left(x_{m}\right)=s^{k}$. And we proceed as above.

- Suppose $x \notin C_{\geq}^{k}$. This case is treated like the previous one.

So, all these contradictions show that $x \in C^{k-1} \cap C^{k}$ and this concludes the proof.

\section{A.6 Proof of Corollary 2}

The strategy of this proof is simple: if we work with the topological closure of the classes, we obtain a structure that is an ordered covering satisfying all 
conditions of Theorem 2. We then obtain an additive representation of this ordered covering. It is clear that it also represents the partition. The details of this proof are omitted because it mimics the proof of Corollary 1.

\section{References}

Ashby, F. G., Maddox, W. T., 1993. Relations between prototype, exemplar and decision bound models of categorization. Journal of Mathematical Psychology 37, 372-400.

Azrieli, Y., Lehrer, E., 2007. Categorization generated by extended prototypes - an axiomatic approach. Journal of Mathematical Psychology $51,14-28$.

Bouyssou, D., Marchant, T., 2007a. An axiomatic approach to noncompensatory sorting methods in MCDM, i: the case of two categories. European Journal of Operational Research 178, 217-245.

Bouyssou, D., Marchant, T., 2007b. An axiomatic approach to noncompensatory sorting methods in MCDM, ii: the general case. European Journal of Operational Research 178, 246-276.

Bouyssou, D., Marchant, T., 2008. XX Unrestricted solvability XX.

Chateauneuf, A., Wakker, P., 1993. From local to global additive representation. Journal of Mathematical Economics 22, 523-545.

Debreu, G., 1960. Topological methods in cardinal utility theory. In: Arrow, K. J., Karlin, S., Suppes, P. (Eds.), Mathematical methods in the Social Sciences. Stanford University Press, Stanford, pp. 16-26.

DeSarbo, W. S., Libby, R., Jedidi, K., 1994. Catscale: A stochastic multidimensional scaling methodology for the spatial analysis of sorting data and the study of stimulus categorization. Computational Statistics \& Data Analysis 18, 165-184.

Fisher, R. A., 1936. The use of multiple measurements in taxonomic problems. Annals of Eugenics 7, 179-188.

Goldstein, W. M., 1991. Decomposable threshold models. Journal of Mathematical Psychology 35, 64-79.

Jacquet-Lagrèze, E., 1995. An application of the UTA discriminant model for the evaluation of sc R\&D projects. In: Pardalos, P., Siskos, Y., Zopounidis, C. (Eds.), Advances in Multicriteria Analysis. Nonconvex Optimization and its Applications. Kluwer Academic, Dordrecht, pp. 203-211.

Krantz, D. H., Luce, R. D., Suppes, P., Tversky, A., 1971. Foundations of measurement: Additive and polynomial representations. Academic Press, London.

McLachlan, G. J., 2004. Discriminant Analysis and Statistical Pattern Recognition. Wiley-Interscience.

Munkres, J. R., 2000. Topology, 2nd Edition. Prentice Hall, Upper Saddle River. 
Nakamura, Y., 2004. Trichotomic preferences for gambles. Journal of Mathematical Psychology 48 (6), 385-398.

Reed, S. K., 1972. Pattern recognition and categorization. Cognitive Psychology $23,91-140$.

Słowiński, R., Greco, S., Matarazzo, B., 2002. Axiomatization of utility, outranking and decision-rule preference models for multiple-criteria classification problems under partial inconsistency with the dominance principle. Control and Cybernetics 31 (4), 1005-1035.

Stanton, R. D., Nosofsky, R. M., Zaki, S. R., 2002. Comparisons between exemplar similarity and mixed prototype models using a linearly separable category structure. Memory and Cognition 30, 934-944.

Vind, K., 1991. Independent preferences. Journal of Mathematical Economics 20, 119-135.

Vind, K., 2003. Independence, Additivity, Uncertainty. Springer, Berlin.

Wakker, P., 1988. The algebraic versus the topological approach to additive representations. Journal of Mathematical Psychology 32, 421-435. 